\title{
Criticality theory for Schrödinger operators on graphs
}

\author{
Matthias Keller Yehuda Pinchover Felix Pogorzelski
}

\begin{abstract}
We study Schrödinger operators given by positive quadratic forms on infinite graphs. From there, we develop a criticality theory for Schrödinger operators on general weighted graphs.
\end{abstract}

Keywords. Green function, Ground state, Positive solutions, Discrete Schrödinger operators, Weighted graphs

\section{Introduction}

Schrödinger operators are an important class of operators in analysis and mathematical physics. One of the first and most fundamental questions in the analysis of positive Schrödinger operators is the question of criticality. In fact, it is the starting point to study Liouville theorems, the large time behaviour of the heat kernel, Hardy inequalities, properties of the ground state as well as basic questions in spectral theory.

Such a theory is classical for second-order linear elliptic operators (not necessarily symmetric) with real coefficients for which we refer the reader to [24, 27, 31] and references therein. See also [29] (and references therein) for recent developments in the quasilinear case, and [36, 37] for the case of generalized Schrödinger forms.

In this paper we present a criticality theory for positive Schrödinger operators on general weighted graphs. First applications of this theory were already obtained in [20] and [2].

While criticality theory was so far not studied for graphs, there is a closely related phenomena called recurrence. This notion appears in the setting of random walks, the situation of positive matrices and in the very general context of Dirichlet forms. In each of these settings there exists a vast body of literature so we mention here only the monograph [42] for random walks, the chapter in the survey [23, Section 6] for positive matrices and [8] for the theory of

M. Keller: Institut für Mathematik, Universität Potsdam, 14476 Potsdam, Germany; e-mail: mkeller@math.uni-potsdam.de

Y. Pinchover and F. Pogorzelski: Department of Mathematics, Technion-Israel Institute of Technology, 32000 Haifa, Israel; e-mail: pincho@technion.ac.il and felixp@technion.ac.il

Mathematics Subject Classification (2010): Primary 39A12; Secondary 31C20, 31C25, 35B09, 35P05, 35R02, 47B39 
Dirichlet forms. We elaborate in depth about the relationship of recurrence and criticality in Remark 5.8 in Section 5 .

The paper is structured as follows. In the following section the basic setting is introduced. In Section 3 we discuss self-adjoint realizations of the formal Laplacian. This is used to prove an Allegretto-Piepenbrink theorem in Section 4 which utilizes a local Harnack inequality and a ground state transform. We proceed in Section 5 by a characterization of criticality and subcriticality. This includes in Section 5.4 a particular discussion of the Green function. We continue to characterize a phenomenon called uniform subcriticality in Section 5.5. Finally, we characterize a phenomenon called null-criticality in Section 6, In particular, we characterize it in terms of the large time behavior of the heat kernel and in terms of the behavior of the Green function near criticality.

\section{Set up}

\subsection{Graphs}

Let $X$ be an infinite set equipped with the discrete topology. A graph over $X$ is a symmetric function $b: X \times X \rightarrow[0, \infty)$ with zero diagonal such that it is locally summable, that is,

$$
\sum_{y \in X} b(x, y)<\infty \quad \text { for all } x \in X .
$$

We call the elements of $X$ vertices. We say that $x, y \in X$ are adjacent or neighbors or connected by an edge if $b(x, y)>0$ in which case we write $x \sim y$. We call $b$ connected if for every $x$ and $y$ in $X$ there are $x_{0}, \ldots, x_{n}$ in $X$ such that $x_{0}=x, x_{n}=y$ and $x_{i} \sim x_{i+1}$ for $i=0, \ldots, n-1$.

\subsection{Formal Schrödinger operators and forms}

Let $W \subseteq X$, we denote by $C(W)$ (resp., $C_{c}(W)$ ) the space of real valued functions on $W$ (resp., with compact support in $W$ ). By extending functions by zero on $X \backslash W$ the space $C(W)$ will be considered as a subspace of $C(X)$.

We write $f \geq c$ (resp., $f=c$ ) in $W$, whenever a function $f \in C(W)$ is larger or equal (resp., equal) to the function which takes the constant value $c \in \mathbb{R}$ on $W$. In particular, with a slight abuse of notation, we do not distinguish between constants and constant functions in notation and may write for example 1 for the function that takes constantly the value 1 on $X$. We say that $f \in C(W)$ is positive in $W$ if $f \geq 0$ and $f \neq 0$ in $W$, in this case, we also use the notation $f \supsetneqq 0$. We use the notation $f=f_{+}-f_{-}$, where $f_{ \pm}:=(0 \vee \pm f)$ are the positive and the negative parts of $f$.

Given a graph $b$ over $X$, we introduce the associated formal Laplacian $L=L_{b}$ acting on the space

$$
F(X):=\left\{f \in C(X)\left|\sum_{y \in X} b(x, y)\right| f(y) \mid<\infty \text { for all } x \in X\right\}
$$


by

$$
L f(x):=\sum_{y \in X} b(x, y)(f(x)-f(y))
$$

By the summability assumption on $b$ we have $\ell^{\infty}(X) \subset F(X)$.

For a potential $q: X \rightarrow \mathbb{R}$, we define the formal Schrödinger operator $H$ on $F(X)$ by

$$
H:=L+q
$$

The associated bilinear form $h$ of $H$ on $C_{c}(X) \times C_{c}(X)$ is given by

$$
h(\varphi, \psi):=\frac{1}{2} \sum_{x, y \in X} b(x, y)(\varphi(x)-\varphi(y))(\psi(x)-\psi(y))+\sum_{x \in X} q(x) \varphi(x) \psi(x) .
$$

We denote by $h(\varphi):=h(\varphi, \varphi)$ the induced quadratic form on $C_{c}(X)$. Furthermore, we write $h \geq 0$ on $C_{c}(X)$ (or in short $h \geq 0$ ) if $h(\varphi) \geq 0$ for all $\varphi \in C_{c}(X)$.

A strictly positive function $m: X \rightarrow(0, \infty)$ extends to a measure via $m(A):=\sum_{x \in A} m(x)$, where $A \subseteq X$. The real Hilbert space $\ell^{2}(X, m)$ is the space of all $m$-square summable functions, equipped with the scalar product

$$
\langle f, g\rangle_{m}:=\sum_{X} f g m:=\sum_{x \in X} f(x) g(x) m(x) \quad f, g \in \ell^{2}(X, m),
$$

and the induced norm $\|\cdot\|=\|\cdot\|_{m}$. Given a measure $m$ we speak of a graph $b$ over $(X, m)$.

With a slight abuse of notation, we keep writing $\ell^{2}(X, m)$ also in the case where $m \supsetneqq 0$. Of a particular importance is the case when $m=1$ is the counting measure. In this case, we denote by $\ell^{2}(X)$ the Hilbert space of square summable functions equipped with the scalar product

$$
\langle f, g\rangle:=\langle f, g\rangle_{1}=\sum_{X} f g=\sum_{x \in X} f(x) g(x) \quad f, g \in \ell^{2}(X) .
$$

There is a Green formula relating $H$ and $h$. The formula follows by a direct algebraic manipulation, where one has to ensure that all the involved sums converge absolutely. This is however a consequence of the Cauchy-Schwarz inequality and Fubini's theorem. For details see [10, Lemma 4.7].

Lemma 2.1 (Green formula). For all $f \in F(X)$ and $\psi \in C_{c}(X)$ one has

$$
\begin{aligned}
\frac{1}{2} \sum_{x, y \in X} b(x, y)(f(x)- & f(y))(\psi(x)-\psi(y))+\sum_{x \in X} q(x) f(x) \psi(x) \\
= & \sum_{x \in X}(H f)(x) \psi(x)=\sum_{x \in X} f(x)(H \psi)(x) .
\end{aligned}
$$

Furthermore, $H C_{c}(X) \subseteq \ell^{2}(X)$, so if $\varphi, \psi \in C_{c}(X)$, then

$$
h(\varphi, \psi)=\langle H \varphi, \psi\rangle=\langle\varphi, H \psi\rangle .
$$

Hence, we can recover $H$ from $h$, so we also speak of $H$ associated to $h$. 


\section{Self-adjoint realization of the operator}

In this section we discuss the closability of the form $h$ in weighted $\ell^{2}$-spaces over $X$. This allows us to define a self-adjoint operator which coincides with $H$ in the case of the counting measure. This opens the door to the use of operator theoretic arguments in the succeeding discussion.

The next theorem states that we can always close a nonnegative form $h$ on $\ell^{2}(X, m)$ for every measure $m$, and that the corresponding selfadjoint operator $H^{(m)}$ acts as $\frac{1}{m} H$ on functions in $F \cap D\left(H^{(m)}\right)$, where $D\left(H^{(m)}\right)$ is the domain of $H^{(m)}$.

We recall that a form $h \geq 0$ on $C_{c}(X)$ is closable in $\ell^{2}(X, m)$ if a Cauchy sequence $\left(\varphi_{n}\right)$ in $C_{c}(X)$ with respect to the form norm $\|\cdot\|_{h, m}:=\left(h(\cdot)+\|\cdot\|_{m}^{2}\right)^{1 / 2}$ such that $\left\|\varphi_{n}\right\|_{m} \rightarrow 0$ satisfies $h\left(\varphi_{n}\right) \rightarrow 0$, as $n \rightarrow \infty$. The closure $h^{(m)}$ is then the extension of $h$ to $\bar{C}_{c}(X) \|^{\|\cdot\|_{h, m}}$.

Recall also that $D\left(H^{(m)}\right)$, the domain of the corresponding selfadjoint operator $H^{(m)}$, is the linear space of all functions $f \in D\left(h^{(m)}\right)$ such that there is $g \in \ell^{2}(X, m)$ so that $h^{(m)}(f, h)=$ $\langle g, h\rangle_{m}$ for all $h \in D\left(h^{(m)}\right)$.

Theorem 3.1. Let $b$ be a graph over $X$ and $q$ be a potential such that $h \geq 0$ on $C_{c}(X)$. Then, $h$ is closable in $\ell^{2}(X, m)$ for each measure $m: X \rightarrow(0, \infty)$. The closure $h^{(m)}$ of $h$ satisfies the first Beurling-Deny criterium, i.e., for all $f \in D\left(h^{(m)}\right)$ we have $|f| \in D\left(h^{(m)}\right)$ and

$$
h^{(m)}(|f|) \leq h^{(m)}(f)
$$

Moreover, the selfadjoint operator $H^{(m)}$ associated with $h^{(m)}$ satisfies for all $f \in D\left(H^{(m)}\right) \cap$ $F(X)$

$$
H^{(m)} f=\frac{1}{m} H f
$$

Proof. The form $h$ is closable in any $\ell^{2}(X, m)$ by [9, Theorem 2.9]. We denote the closure by $h^{(m)}$.

We show next that the inequality

$$
h(|\varphi|) \leq h(\varphi), \quad \varphi \in C_{c}(X),
$$

extends to functions $f \in D\left(h^{(m)}\right)$. Let $f \in D\left(h^{(m)}\right)$ and let $\left(\varphi_{n}\right)$ be a sequence in $C_{c}(X)$ that converges to $f$ with respect to with respect to the form norm

$$
\|\cdot\|_{h^{(m)}}:=\sqrt{h^{(m)}(\cdot)+\|\cdot\|_{m}^{2}} .
$$

Then,

$$
h^{(m)}\left(\left|\varphi_{n}\right|\right)=h\left(\left|\varphi_{n}\right|\right) \leq h\left(\varphi_{n}\right)=h^{(m)}\left(\varphi_{n}\right) \rightarrow h^{(m)}(f) .
$$

Hence, the sequence $\left(h^{(m)}\left(\left|\varphi_{n}\right|\right)\right)$ is bounded and $\left(\left|\varphi_{n}\right|\right)$ converges to $|f|$ in $\ell^{2}(X, m)$ and, therefore, also pointwise. Since $h^{(m)}$ is closed, it is lower semicontinuous. Hence,

$$
h^{(m)}(|f|) \leq \liminf _{n \rightarrow \infty} h^{(m)}\left(\left|\varphi_{n}\right|\right)<\infty
$$


which yields $|f| \in D\left(h^{(m)}\right)$ and the inequality

$$
h^{(m)}(|f|) \leq h^{(m)}(f)
$$

Now, let $H^{(m)}$ be the selfadjoint operator associated to the closure $h^{(m)}$ in $\ell^{2}(X, m)$. Let $f \in D\left(H^{(m)}\right) \cap F(X)$. Using Lemma 3.2 below, assume that there exist $f_{n} \in C_{c}(X)$ such that $\left|f_{n}\right| \leq|f|$, and $\left(f_{n}\right)$ converges to $f$ with respect to the form norm $\|\cdot\|_{h^{(m)}}$. Then, by the Green formula we have with $\delta_{x}:=1_{\{x\}} / m(x)$

$$
h^{(m)}\left(f_{n}, \delta_{x}\right)=h\left(f_{n}, \delta_{x}\right)=\left\langle H f_{n}, \delta_{x}\right\rangle_{1}=\frac{1}{m(x)} H f_{n}(x)
$$

Again, we use the fact that $\ell^{2}$-convergence on a discrete space implies pointwise convergence. Thus, by Lebesgue's dominated convergence theorem, with $f \in F(X)$ and $\left|f_{n}\right| \leq|f|$, we get

$$
\begin{aligned}
\lim _{n \rightarrow \infty} \frac{1}{m} H f_{n}(x) & =\frac{f(x)}{m(x)}\left(\sum_{y \in X} b(x, y)+q(x)\right)-\lim _{n \rightarrow \infty}\left(\frac{1}{m(x)} \sum_{y \in X} b(x, y) f_{n}(y)\right) \\
& =\frac{1}{m(x)} H f(x) .
\end{aligned}
$$

Lemma 3.2. Let b be a graph over $X$ and assume that $\bar{h}$ is a quadratic form which is closed in $\ell^{2}(X, m)$ for some measure $m: X \rightarrow(0, \infty)$. Then, for every $f \in D(\bar{h}) \cap F(X)$, there exists a sequence $\left(f_{n}\right)$ in $C_{c}(X)$ such that $\left|f_{n}\right| \leq|f|$ for all $n \in \mathbb{N}$ and $f_{n} \rightarrow f$ in $\|\cdot\|_{\bar{h}}$.

Proof. By definition of a closed form $\bar{h}$, there exists a sequence $\left(g_{n}\right) \subset C_{c}(X)$ such that $g_{n} \rightarrow f$ with respect $\|\cdot\|_{h}$. By decomposing $f$ and $g_{n}$ into positive and negative part, it suffices to consider $f \geq 0$ and $g_{n} \geq 0$. Set $f_{n}:=g_{n} \wedge f=\left(f+g_{n}-\left|f-g_{n}\right|\right) / 2$. Since $\bar{h}(|\varphi|) \leq \bar{h}(\varphi)$ for all $\varphi \in D(\bar{h})$, we obtain

$$
\begin{aligned}
& \bar{h}\left(f-f_{n}\right)=\frac{1}{4}\left(\bar{h}\left(f-g_{n}\right)+2 \bar{h}\left(f-g_{n},\left|f-g_{n}\right|\right)+\bar{h}\left(\left|f-g_{n}\right|\right)\right) \\
& \quad \leq \frac{1}{4}\left(\bar{h}\left(f-g_{n}\right)+2 \bar{h}\left(f-g_{n}\right)^{\frac{1}{2}} \bar{h}\left(\left|f-g_{n}\right|\right)^{\frac{1}{2}}+\bar{h}\left(\left|f-g_{n}\right|\right)\right) \leq \bar{h}\left(f-g_{n}\right) .
\end{aligned}
$$

Similarly, $\left\|f_{n}-f\right\|_{m} \leq\left\|g_{n}-f\right\|_{m}$. Hence, $\left\|f_{n}-f\right\|_{\bar{h}} \leq\left\|g_{n}-f\right\|_{\bar{h}}$. Thus, the positive and negative parts of $f_{n}$ converge to $f_{ \pm}$, and this finishes the proof.

Remark 3.3. One can characterize all positive closed forms on discrete sets that have $C_{c}(X)$ as a core and that satisfy the second Beurling-Deny criterion, i.e., $h(f \vee 1) \leq h(f)$ for all $f$ in the domain of $h$, see [17]. Those forms are exactly closures of a form $h$ on $C_{c}(X)$ as above associated with a graph $b$ and a potential $q \geq 0$. So, it is natural to ask the question whether our setting actually covers all positive closed forms that satisfy the first Beurling-Deny criterion

$$
h(|\varphi|) \leq h(\varphi), \quad \varphi \in C_{c}(X) .
$$

and have $C_{c}(X)$ as a core. This is however not the case as the following example shows: 
Let $X=\mathbb{N}_{0}$ and let $h$ be a form on $C_{c}(X)$ acting as

$$
h(\varphi)=\sum_{k \in \mathbb{N}_{0}} \varphi(k)^{2}-\sum_{k \in \mathbb{N}} \frac{1}{k} \varphi(0) \varphi(k), \quad \varphi \in C_{c}(X) .
$$

It is not hard to see that $h \geq 0$ : If $\varphi(0)=0$, then clearly $h(\varphi) \geq 0$, so assume $\varphi(0)=1$. Then $h(\varphi)=1+\sum_{k \in \mathbb{N}} \varphi(k)(\varphi(k)-1 / k)$. So minimizing each term in the sum $\varphi(k)(\varphi(k)-1 / k)$ yields that the minimum is assumed for the function $\varphi_{0}$ such that $\varphi_{0}(k)=1 / 2 k, k \geq 1$ and $\varphi_{0}(0)=1$. However, $h\left(\varphi_{0}\right)=1-\sum_{k \in \mathbb{N}} 1 /(2 k)^{2}=1-\pi^{2} / 24 \geq 0$. Furthermore, an immediate calculation yields that the associated bilinear form

$$
h(\varphi, \psi)=\sum_{k \in \mathbb{N}_{0}} \varphi(k) \psi(k)-\frac{1}{2} \sum_{k \in \mathbb{N}} \frac{1}{k}(\varphi(0) \psi(k)+\varphi(k) \psi(0)), \quad \varphi \in C_{c}(X) .
$$

satisfies $h\left(1_{k}, 1_{l}\right) \leq 0, k \neq l$. This, however, can easily be seen to be equivalent to the first Beurling-Deny criterion.

Moreover, taking the counting measure one easily sees that every $\ell^{2}\left(\mathbb{N}_{0}\right)$-sequence $\left(\varphi_{n}\right)$ converging to zero satisfies $h\left(\varphi_{n}\right) \rightarrow 0$ since $|h(\varphi)| \leq\left(1+\pi^{2} / 6\right)\|\varphi\|^{2}$ for all $\varphi \in C_{c}(X)$. Hence, $h$ is closable in $\ell^{2}\left(\mathbb{N}_{0}\right)$. On the other hand, it is also obvious that one can not write $h$ as a form associated to a graph $b$ and a potential $q$ as above (indeed, one would get the difference of two divergent sums).

Returning to the operator $H^{(m)}$ from above, we denote its spectrum by $\sigma\left(H^{(m)}\right)$. The well known Laplace transform of $H^{(m)}$ is used to related the resolvent and the semigroup.

Lemma 3.4 (Laplace transform). Let b be a graph over $X$, let $q$ be a potential such that $h \geq 0$ on $C_{c}(X)$ and let $m$ be a measure of full support on $X$. For all $\lambda<\inf \sigma\left(H^{(m)}\right)$ on $\ell^{2}(X, m)$ and $f \in \ell^{2}(X, m)$

$$
\left(H^{(m)}-\lambda\right)^{-1} f=\int_{0}^{\infty} \mathrm{e}^{t \lambda} \mathrm{e}^{-t H^{(m)}} f \mathrm{~d} t .
$$

Proof. This follows from the spectral theorem.

With the help of this lemma we remark the following fact for the resolvent of $H^{(m)}$.

Corollary 3.5. Let $b$ be a graph over $X$ and $q$ be a potential such that $h \geq 0$ on $C_{c}(X)$. Then, for any measure $m: X \rightarrow(0, \infty)$ the resolvent of the operator $H^{(m)}$ is positivity preserving, i.e. for any $f \in \ell^{2}(X, m)$ with $f \geq 0$ we have $\left(H^{(m)}-\lambda\right)^{-1} f \geq 0$ for all $\lambda<\inf \sigma\left(H^{(m)}\right)$.

Proof. Positivity preservation of the semigroup $\mathrm{e}^{-t H^{(m)}}$ follows from the first Beurling-Deny criterium proven above, see [33, Theorem XIII.50]. Hence, positivity preservation of the resolvent follows from the Laplace transform.

To study the spectrum of the operator $H^{(m)}$ via solutions it is essential to know the action of $H^{(m)}$ explicitly. The theorem above (Theorem 3.1) only guarantees this knowledge for functions in $D\left(H^{(m)}\right) \cap F(X)$. 
The following proposition provides us with two criteria to ensure that $D\left(H^{(m)}\right) \subseteq F(X)$. The first is on the Laplacian part $L$ of $H$ and the second on the potential $q$. Morally, the assumption on $L$ implies $\ell^{2} \subseteq F(X)$ and the assumption on $q$ implies that the functions of finite energy are included in $F(X)$. The statements can all be extracted from the considerations in [9] and [16]. However, we include a short proof for the convenience of the reader.

Proposition 3.6. Let a graph b over a discrete measure space $(X, m)$ be given, and let $q: X \rightarrow$ $\mathbb{R}$ be such that $h \geq 0$ on $C_{c}(X)$. Then, $D\left(H^{(m)}\right) \subseteq F(X)$ if one of the following assumptions is satisfied:

(a) $\frac{1}{m} L C_{c}(X) \subseteq \ell^{2}(X, m)$. This condition is in particular satisfied if one of the following assumptions holds:

(a1) The graph b is locally finite.

(a2) The measure $m$ satisfies $\inf _{x \in X} m(x)>0$.

(a3) The function $\operatorname{Deg}_{m}(x):=\frac{1}{m(x)} \sum_{y \in X} b(x, y)$ is bounded on $X$.

(b) For some $\varepsilon>0$ we have $h(\varphi) \geq \varepsilon\left\langle q_{-} \varphi, \varphi\right\rangle_{1}$ for all $\varphi \in C_{c}(X)$.

Proof. (a) By definition of $H^{(m)}$ we have $D\left(H^{(m)}\right) \subseteq \ell^{2}(X, m)$, and we show $\ell^{2}(X, m) \subseteq$ $F(X)$ under the assumption (a). By considering characteristic functions of vertices one finds that $\frac{1}{m} L C_{c}(X) \subseteq \ell^{2}(X, m)$ is equivalent to the fact that the functions $\varphi_{x}=b(x, \cdot) / m(\cdot)$, $x \in X$, are in $\ell^{2}(X, m)$. Hence, for $f \in \ell^{2}(X, m)$, we have by the Cauchy-Schwarz inequality

$$
\sum_{y \in X} b(x, y)|f(y)|=\left\langle\varphi_{x},|f|\right\rangle_{m} \leq\left\|\varphi_{x}\right\|_{m}\|f\|_{m} .
$$

Thus, $f \in F(X)$ and therefore, $D\left(H^{(m)}\right) \subseteq \ell^{2}(X, m) \subseteq F(X)$. The "in particular" statements follow easily from the characterization of $\frac{1}{m} L C_{c}(X) \subseteq \ell^{2}(X, m)$ by $\varphi_{x} \in \ell^{2}(X, m), x \in X$.

Assume $h_{+}(\varphi):=\left\langle\left(L+q_{+}\right) \varphi, \varphi\right\rangle_{1} \geq(1+\varepsilon)\left\langle q_{-} \varphi, \varphi\right\rangle_{1}$, for all $\varphi \in C_{c}(X)$. By standard perturbation theory, the domain $D\left(h_{+, m}\right)$ of the closure $h_{+, m}=\bar{h}_{+}$of the quadratic form $h_{+}$on $\ell^{2}(X, m)$ is equal to the domain $D\left(h^{(m)}\right)$ of $h^{(m)}=\bar{h}$. (Recall that $h$ is closable in $\ell^{2}(X, m)$ by Theorem 3.1.) From standard arguments using Fatou's lemma it follows (cf. [16]) that

$$
h_{+, m}(f, f)=\frac{1}{2} \sum_{x, y \in X} b(x, y)(f(x)-f(y))^{2}+\sum_{x \in X} q_{+}(x) f(x)^{2}
$$

for $f \in D\left(h_{+, m}\right)$, and therefore,

$$
D\left(h_{+, m}\right) \subseteq \widetilde{D}:=\left\{f \in C(X) \mid \widetilde{h}(f):=\frac{1}{2} \sum_{x, y \in X} b(x, y)(f(x)-f(y))^{2}<\infty\right\} .
$$

So, one is left to check $\widetilde{D} \subseteq F(X)$ which can be seen via the inequalities

$$
\begin{aligned}
\sum_{y \in X} b(x, y)|f(y)| & \leq \sum_{y \in X} b(x, y)|f(x)-f(y)|+|f(x)| B(x) \\
& \leq \sqrt{2} B(x)^{1 / 2} \widetilde{h}(f)^{1 / 2}+|f(x)| B(x),
\end{aligned}
$$

where $B(x):=\sum_{y \in X} b(x, y)<\infty$ by our assumption. 
Remark 3.7. Condition (a) of Proposition 3.6 is equivalent to the condition $C_{c}(X) \subseteq D\left(H^{(m)}\right)$ which can be directly read from the Green formula, Lemma 2.1, and the abstract definition of $D\left(H^{(m)}\right)$. Furthermore, (a2) implies that

$$
D\left(H^{(m)}\right)=\left\{f \in \ell^{2}(X, m) \mid H f \in \ell^{2}(X, m)\right\},
$$

whenever $q \geq 0$, see [16]. Finally, (a3) implies that the operator $H^{(m)}$ is bounded whenever $q$ is bounded, see [11, Theorem 9.3].

We next draw a corollary from Corollary 3.5 and Proposition 3.6 .

Corollary 3.8 (Action of $\left.H^{(m)}\right)$. Let a graph b over a discrete measure space $(X, m)$ be given, and let $q: X \rightarrow \mathbb{R}$ be such that $h \geq 0$. Assume one of the conditions in Proposition 3.6 holds. Then, for all $f \in D\left(H^{(m)}\right)$

$$
H^{(m)} f=\frac{1}{m} H f .
$$

In particular, for $\lambda<0$ and $g \in \ell^{2}(X, m)$, the function $u_{\lambda}:=\left(H^{(m)}-\lambda\right)^{-1} g$ is in $F(X)$. Moreover, if $g \geq 0$, then $u_{\lambda}$ is a positive solution of the equation

$$
(H-\lambda m) u_{\lambda}=g \quad \text { in } X .
$$

\section{Allegretto-Piepenbrink theorem}

Let $\lambda_{0}\left(H^{(m)}\right)$ and $\lambda_{0}^{\text {ess }}\left(H^{(m)}\right)$ be the bottom of the spectrum and the bottom of the essential spectrum of the selfadjoint operator $H^{(m)}$. In this subsection we prove an Allegretto-Piepenbrinktype theorem relating $\lambda_{0}\left(H^{(m)}\right)$ and $\lambda_{0}^{\text {ess }}\left(H^{(m)}\right)$ to the existence of positive (super)solutions of the equation $(H-\lambda m) u=0$.

For $q \geq 0$ and $\lambda_{0}\left(H^{(m)}\right)$ such a result is found in [10], and for $\lambda_{0}^{\text {ess }}\left(H^{(m)}\right)$ see [15]. For $q=0$ and locally finite graphs see [3], and for Dirichlet forms see [5, 22].

Definition 4.1 ((Super)harmonic function). We say that a function $u$ is $H$-(super)harmonic on $W \subseteq X$ if $u \in F(X)$ and $H u=0,(H u \geq 0)$ on $W$. We write

$$
H \geq 0 \quad \text { on } W
$$

if there exists a positive $H$-superharmonic function $u$ on $W$.

Theorem 4.2 (Allegretto-Piepenbrink-type theorem). Let b be a connected infinite graph over $X$ and $q$ be a potential such that $h \geq 0$ on $C_{c}(X)$. Let $m$ be a given measure $m: X \rightarrow(0, \infty)$ over $X$.

(a) We have

$$
\lambda_{0}\left(H^{(m)}\right) \geq \sup \{\lambda \in \mathbb{R} \mid(H-\lambda m) \geq 0 \text { on } X\} .
$$


(b) In addition, if $D\left(H^{(m)}\right) \subseteq F(X)$, then

$$
\lambda_{0}\left(H^{(m)}\right)=\max \{\lambda \in \mathbb{R} \mid(H-\lambda m) \geq 0 \text { on } X\} .
$$

(c) Moreover, if $\frac{1}{m} L C_{c}(X) \subseteq \ell^{2}(X, m)$, then

$$
\lambda_{0}^{\text {ess }}\left(H^{(m)}\right)=\sup \{\lambda \in \mathbb{R} \mid(H-\lambda m) \geq 0 \text { on } X \backslash K \text { for a finite } K \subset X\} .
$$

Remark 4.3. Clearly, on finite graphs there are no positive $(H-\lambda)$-harmonic functions for $\lambda<\lambda_{0}\left(H^{(m)}\right)$ but only $(H-\lambda)$-superharmonic functions. In [10] an example is given that shows that in the non locally finite setting there might be only $(H-\lambda)$-superharmonic functions for $\lambda<\lambda_{0}\left(H^{(m)}\right)$ either.

Remark 4.4. We formulated Theorem 4.2 for the case $h \geq 0$. By fixing the measure $m$ before choosing the potential $q$, one can allow for a potential $q$ such the resulting form on $C_{c}(X)$ is form bounded from below in $\ell^{2}(X, m)$. Such forms are also closable (cf. [9, Theorem 2.9]) and the arguments carry over verbatim with the obvious adaption made.

The proof of the Allegretto-Piepenbrink theorem uses a local Harnack inequality and a ground state transform which are proven next.

\subsection{A local Harnack inequality}

The following local Harnack inequality is a slightly more general formulation of [10, Proposition 3.4] (for earlier versions see e.g. [4, 43]). However, the proof can be carried over verbatim, but as it is short we give it here for the sake of our paper being self-contained.

Lemma 4.5 (Harnack inequality). Let $W \subseteq X$ be a connected and finite set and let $f \in C(X)$. There exists a positive constant $C=C(H, W, f)$ such that for any nonnegative function $u \in$ $F(X)$ satisfying $(H-f) u \geq 0$ on $W$, the following inequality holds

$$
\max _{W} u \leq C \min _{W} u
$$

In particular, any positive $H$-superharmonic function is strictly positive.

Furthermore, the above Harnack constant $C(H, W, f)$ can be chosen such that for any $f \leq$ $\tilde{f}$ we have

$$
C(H, W, \tilde{f}) \leq C(H, W, f) .
$$

Proof. We may assume that $u \supsetneqq 0$ on $W$. Let

$$
d(x):=\sum_{y \in X} b(x, y)+q(x)-f(x), \quad x \in X .
$$

Take $y_{0} \in W$ with $u\left(y_{0}\right)>0$. Then, the inequality $H u \geq f u$ gives for all $x \sim y_{0}$

$$
d(x) u(x) \geq \sum_{y \in X} b(x, y) u(y) \geq b\left(x, y_{0}\right) u\left(y_{0}\right) .
$$


We conclude that $d$ and $u$ are strictly positive for all $x \sim y_{0}$. Since $W$ is connected, $d$ and $u$ are strictly positive on $W$. Let a path $x_{0} \sim \ldots \sim x_{n}$ in $W$ from the maximum of $u$ at $x_{\max }=x_{0}$ to the minimum at $x_{\min }=x_{n}$ be given. We obtain

$$
u\left(x_{\max }\right) \leq u\left(x_{\min }\right) \prod_{j=0}^{n-1} \frac{d\left(x_{j}\right)}{b\left(x_{j}, x_{j+1}\right)} .
$$

Taking the minimum of the product on the right side (over all possible paths in $W$ ) yields the constant $C(H, W, f)$. The monotonicity in $f$ follows as $\tilde{d} \leq d$ whenever $f \leq \tilde{f}$ for $\tilde{d}$ defined with $\tilde{f}$.

A graph $b$ over $X$ is called locally finite if for all $x \in X$

$$
\#\{y \in X \mid b(x, y)>0\}<\infty
$$

The following Harnack principle is an immediate consequence of the local Harnack inequality and Fatou's lemma.

Lemma 4.6 (Harnack principle). Let the graph be a connected and let $f \in C(X)$. Let $\left(u_{n}\right)$ be a sequence of positive functions in $F(X)$ such that $H u_{n} \geq f u_{n}$ in $X$, and suppose that there is $o \in X$ such that $C^{-1} \leq u_{n}(o) \leq C$ for some $C>0$. Then, there exists a subsequence $\left(u_{n_{k}}\right)$ that converges pointwise to a strictly positive function $u \in F(X)$ such that $H u \geq f u$.

Furthermore, assume that one of the following properties hold true:

- The graph b is locally finite.

- $\left(u_{n_{k}}\right)$ is monotone increasing in $k$.

- There exists $g \in F(X)$ such that $u_{n_{k}} \leq$ for all $k \in \mathbb{N}$ in $X$.

Then, $H u_{n_{k}} \rightarrow H u$.

Proof. By the Harnack inequality the set of positive functions $v$ such that $H v \geq f v$ in $X$ such that $C^{-1} \leq v(o) \leq C$ for some fixed $o \in X$ and a positive constant $C>0$ is compact with respect to the product topology, that is with respect to pointwise convergence. Hence, the sequence $\left(u_{n}\right)$ has a convergent subsequence $\left(u_{n_{k}}\right)$ that converges to a strictly positive limiting function $u$. We are left to check that $u$ satisfies $H u \geq f u$. Since the functions $u_{n}$ satisfy $H u \geq$ $f u$, we have for all $x \in X$

$$
\sum_{y \in X} b(x, y) u_{n_{k}}(y) \leq u_{n_{k}}(x)\left(\sum_{y \in X} b(x, y)+q(x)-f(x)\right) .
$$

By Fatou's lemma we have

$$
\sum_{y \in X} b(x, y) u(y) \leq \liminf _{k \rightarrow \infty} \sum_{y \in X} b(x, y) u_{n_{k}}(y) \leq u(x)\left(\sum_{y \in X} b(x, y)+q(x)-f(x)\right)
$$


Thus, $u \in F(X)$ and $H u \geq f u$.

If the graph is locally finite, then all involved sums are over finitely many terms only. Therefore, we can interchange the sum with the limit. On the other hand, if $u_{n_{k}}(y), y \in X$, are monotone increasing in $k$ (resp., $u_{n_{k}} \leq g \in F$ ), then we can apply instead of Fatou, the monotone convergence theorem of Beppo-Levi (resp., the dominated convergence theorem), to get the convergence $H u_{n_{k}} \rightarrow H u$.

\subsection{The ground state transform}

The ground state transform uses a positive harmonic function to turn Schrödinger operators into Laplace-type operators.

For $v \in C(X)$, define the operator $T_{v}: C(X) \rightarrow C(X)$ as

$$
T_{v} f:=v f
$$

For strictly positive $v$ the operator $T_{v}$ is bijective and $T_{v}^{-1}=T_{v^{-1}}$. Given a strictly positive $v$, we define the operator $H_{v}$ on $F_{v}(X):=T_{v}^{-1} F(X)$ by

$$
H_{v}:=T_{v}^{-1} H T_{v}
$$

For $v \in F(X)$ one immediately checks that the constant function belongs to $F_{v}(X)$ since $1=T_{v}^{-1} v$. By a direct computation we obtain

$$
H_{v} f(x)=\frac{1}{v(x)^{2}} \sum_{y \in X} b(x, y) v(y) v(x)(f(x)-f(y))+\frac{(H v)(x)}{v(x)} f(x), \quad f \in F_{v}(X) .
$$

If in addition, $v$ is $H$-harmonic (resp., $H$-superharmonic) in $X$, then

$$
H_{v} 1=H v=0
$$

(resp., $\left.H_{v} 1 \geq 0\right)$ in $X$. In this case, the operator $H_{v}$ is called a ground state transform of the operator $H$ with respect to $v$.

We start with the following observation.

Lemma 4.7. Let $u$ be a $H$-(super)harmonic function and let $v$ be a non-vanishing positive function. Then the function $u / v$ is a $H_{v}$-(super)harmonic function.

Proof. We calculate straightforwardly that $H_{v}(u / v)=v^{-1} H u \geq 0$.

For $0 \supsetneqq v \in F(X)$, we define the bilinear form $h_{v}: C_{c}(X) \times C_{c}(X) \rightarrow \mathbb{R}$ via

$$
h_{v}(\varphi, \psi):=\frac{1}{2} \sum_{x, y \in X} b(x, y) v(x) v(y)(\varphi(x)-\varphi(y))(\psi(x)-\psi(y)) .
$$

Using the Cauchy-Schwarz inequality one immediately sees that the right hand side converges by the virtue of $v \in F(X)$ and $\sum_{y} b(x, y)<\infty, x \in X$, for all $\varphi, \psi \in C_{c}(X)$. Furthermore, 
we denote the induced quadratic form also by $h_{v}$. Whenever $v$ is a $H$-(super)harmonic positive function we call $h_{v}$ a ground state transform of $h$. This terminology is justified by the following proposition. There the relation between $H_{v}, h_{v}$ and $H, h$, for solutions $v$ of a equation $H v=f v$ is discussed. Indeed, a ground state of $H$ solves such an equation in the case $f=0$. This is a well known fact; see e.g. [3, 6, 7, 5, 10, 38] for proofs in (closely) related contexts.

Proposition 4.8 (Ground state transform). Let $v \in F(X)$ be strictly positive, $f \in C(X)$ such that $H v=f v$. Then

$$
h(\varphi, \psi)=h_{v}\left(\frac{\varphi}{v}, \frac{\psi}{v}\right)+\langle f \varphi, \psi\rangle_{1} \quad \varphi, \psi \in C_{c}(X)
$$

$H_{v} \varphi \in \ell^{2}\left(X, v^{2}\right)$ for $\varphi \in C_{c}(X)$, and

$$
h_{v}(\varphi, \psi)=\left\langle H_{v} \varphi, \psi\right\rangle_{v^{2}}-\langle f \varphi, \psi\rangle_{v^{2}} \quad \varphi, \psi \in C_{c}(X),
$$

where $\langle\cdot, \cdot\rangle_{v^{2}}$ is the scalar product of $\ell^{2}\left(X, v^{2}\right)$.

Proof. The first formula follows by a direct computation (cf. [5, Theorem 10.1] and [10, Proposition 3.2]). For the second formula, note that $H_{v} \varphi \in \ell^{2}\left(X, v^{2}\right), \varphi \in C_{c}(X)$, is equivalent to $H_{v} 1_{\{x\}} \in \ell^{2}\left(X, v^{2}\right), x \in X$, which is equivalent to the functions $y \mapsto b(x, y) / v(x)$ being in $\ell^{2}\left(X, v^{2}\right), x \in X$. However, this follows from $\sum_{y \in X} b(x, y)<\infty, x \in X$. Furthermore, observe that by the Green formula

$$
h_{v}(\varphi, \psi)=h\left(T_{v} \varphi, T_{v} \psi\right)-\langle f \varphi, \psi\rangle_{v^{2}}=\left\langle H_{v} \varphi, \psi\right\rangle_{v^{2}}-\langle f \varphi, \psi\rangle_{v^{2}}
$$

Remark 4.9. A particular advantage of the ground state transform $h_{v}$ with respect to a strictly positive $H$-harmonic function $v$ is that it is Markovian (or has the Markov property), that is,

$$
h_{v}(0 \vee \varphi \wedge 1) \leq h_{v}(\varphi), \quad \varphi \in C_{c}(X)
$$

while for $h$ one only has

$$
h(|\varphi|) \leq h(\varphi), \quad \varphi \in C_{c}(X)
$$

By decomposing a function into its positive and negative part one sees that the inequality for the modulus can be deduced from the Markov property.

\subsection{Proof of the Allegretto-Piepenbrink theorem}

Proof. The inequalities " $\geq$ " in (a) and (b) follow directly from the ground state transform applied to $L+q-\lambda m$.

Let us turn to the inequality " $\leq$ " in (b) when the "max" is replaced by a "sup". So, fix a vertex $x \in X$. Notice that for any $\lambda<\lambda_{0}\left(H^{(m)}\right)$ and $\delta_{x}:=1_{\{x\}} / m(x)$, the functions

$$
u_{\lambda}=\left(H^{(m)}-\lambda\right)^{-1} \delta_{x}
$$


are in $D\left(H^{(m)}\right)$. Moreover, $u_{\lambda}$ are positive by Corollary 3.5. As $H^{(m)}=\frac{1}{m} H$ on $D\left(H^{(m)}\right) \cap$ $F(X)$, and $D\left(H^{(m)}\right) \subseteq F(X)$ by assumption, we infer by Corollary 3.8

$$
(H-\lambda m) u_{\lambda}=m\left(H^{(m)}-\lambda\right) u_{\lambda}=m(x) \delta_{x}=1_{\{x\}} \geq 0 .
$$

Furthermore, by the local Harnack inequality (Lemma4.5), the functions $u_{\lambda}$ are strictly positive.

Next we show that in (b) the "sup" is in fact a "max". To show this let

$$
\lambda^{\prime} \leq \lambda<\lambda_{0}\left(H^{(m)}\right) \quad \text { and } \quad g_{\lambda}:=u_{\lambda} / u_{\lambda}(x)
$$

For $y \in X$, let $W_{y}$ be a connected and finite set such that $x, y \in W_{y}$. Then, by the local Harnack inequality, Lemma 4.5, and in particular by the monotonicity of the local Harnack constant with respect to $\lambda$, we have $g_{\lambda}(y) \leq C_{y}$ for all $y \in X$, where $C_{y}=C\left(H, W_{y}, \lambda^{\prime}\right)$. Hence, there is a sequence $\lambda_{n} \rightarrow \lambda_{0}=\lambda_{0}\left(H^{(m)}\right)$ such that $\left(g_{\lambda_{n}}\right)$ converges pointwise to a limit $0 \leq g \in C(X)$. By $\left(H-\lambda_{n} m\right) g_{\lambda_{n}} \geq 0$, we have for all $y \in X$

$$
\sum_{z \in X} b(y, z) g_{\lambda_{n}}(z) \leq g_{\lambda_{n}}(y)\left(\sum_{z \in X} b(y, z)+q(y)-\lambda_{n} m\right) .
$$

The right hand side converges and, therefore, by Fatou's lemma $g \in F(X)$ and $\left(H-\lambda_{0} m\right) g \geq 0$.

Let us turn to the statement (c) concerning the essential spectrum. Recall that by Proposition 3.6, the assumption $\frac{1}{m} L C_{c}(X) \subseteq \ell^{2}(X, m)$ implies that $D\left(H^{(m)}\right) \subseteq F(X)$.

For $W \subseteq X$, let $h_{W}$ be the form acting as

$$
h_{W}(\varphi):=h\left(1_{W} \varphi\right), \quad \varphi \in C_{c}(X) .
$$

Furthermore, let

$$
\partial W:=W \times(X \backslash W)
$$

By Lemma4.10 below, the operator associated to the closure of the form $\left(h-h_{X \backslash K}\right)$ in $\ell^{2}(X, m)$ is compact whenever $\frac{1}{m} L C_{c}(X) \subseteq \ell^{2}(X, m)$ and $K \subseteq X$ is finite. Thus, the operator $H_{X \backslash K}^{(m)}$ associated to $h_{X \backslash K}$ is a compact perturbation of $H^{(m)}$. Hence,

$$
\lambda_{0}\left(H_{X \backslash K}^{(m)}\right) \leq \lambda_{0}^{\mathrm{ess}}\left(H_{X \backslash K}^{(m)}\right)=\lambda_{0}^{\mathrm{ess}}\left(H^{(m)}\right) .
$$

Combining this fact with a Persson-type theorem, see e.g. [12, Proposition 2.1], one finds that

$$
\sup _{K \subset X \text { finite }} \lambda_{0}\left(H_{X \backslash K}^{(m)}\right)=\lambda_{0}^{\text {ess }}\left(H^{(m)}\right) .
$$

Now, the theorem follows by applying (b) to $H_{(X \backslash K)}^{(m)}$.

Lemma 4.10. Let $b$ be a graph over $(X, m)$, let $q$ be a potential and let $K \subseteq X$ be a finite set. The form $\left(h-h_{X \backslash K}\right)$ acts on $C_{c}(X)$ as

$$
\left(h-h_{X \backslash K}\right)(f)=h_{K}(f)-\sum_{(x, y) \in \partial K} b(x, y) f(x) f(y) .
$$

If $\frac{1}{m} L C_{c}(K) \subseteq \ell^{2}(X, m)$, then $\left(h-h_{X \backslash K}\right)$ is bounded on $\ell^{2}(X, m)$, and the associated selfadjoint operator is compact. 
Proof. The formula for $\left(h-h_{X \backslash K}\right)$ follows by a direct computation. For finite $K$, the form $h_{K}$ is zero outside of the finite dimensional space $C_{c}(K)$, and therefore, bounded. The assumption $\frac{1}{m} L C_{c}(K) \subseteq \ell^{2}(X, m)$ is equivalent to the functions $\varphi_{x}: X \rightarrow[0, \infty), y \mapsto b(x, y) / m(y)$ being in $\ell^{2}(X, m)$ for every $x \in K$. So, for $f \in C_{c}(X)$ with $\|f\|_{m}=1$ we have

$$
\begin{aligned}
\left|\sum_{(x, y) \in \partial K,} b(x, y) f(x) f(y)\right| & \leq\left\|f 1_{K}\right\|_{\infty}\left|\sum_{(x, y) \in \partial K} b(x, y) f(y)\right| \\
& \leq\left\|f 1_{K}\right\|_{\infty} \sum_{x \in K}\left\langle\varphi_{x},|f|\right\rangle_{m} \\
& \leq \max _{x \in K} \frac{1}{m(x)} \max _{x \in K}\left\|\varphi_{x}\right\|_{m} \# K
\end{aligned}
$$

Hence, the form $\left(h-h_{X \backslash K}\right)$ is bounded. We can associate a self-adjoint operator $T_{K}$ to the closure of $\left(h-h_{X \backslash K}\right)$ on $\ell^{2}(X, m)$ and let $H_{K}^{(m)}$ be the finite dimensional operator associated to the closure of $h_{K}$ on $\ell^{2}(X, m)$. Set $P_{K}:=T_{K}-H_{K}^{(m)}$. Clearly, $T_{K}$ is compact if $P_{K}$ is compact. Observe that $P_{K}$ acts as

$$
P_{K} f(x)=\frac{1}{m(x)} \sum_{(x, y) \in \partial K} b(x, y) f(y), \quad x \in X .
$$

Let $\left(f_{n}\right)$ be a normalized sequence that converges weakly in $\ell^{2}(X, m)$ to 0 . Then, again using $\varphi_{x} \in \ell^{2}(X, m)$ and the finiteness of $K$, we get

$$
\left\|P_{K} f_{n}\right\|_{m}^{2}=\sum_{x \in K}\left(\frac{1}{m(x)} \sum_{(x, y) \in \partial K} b(x, y) f_{n}(y)\right)^{2} m(x) \leq \# K \max _{x \in K} \frac{\left\langle\varphi_{x}, f_{n}\right\rangle_{m}^{2}}{m(x)} \underset{n \rightarrow \infty}{\longrightarrow} 0 .
$$

Thus, $P_{K}$ is compact and so is $T_{K}$ which finishes the proof.

The following corollary is a "measure-free" version of the Allegretto-Piepenbrink theorem.

Corollary 4.11 (Allegretto-Piepenbrink theorem - measure free version). Let $b$ be a connected graph over $X$ and let $q$ be a potential such that $h \geq 0$. Then,

$$
\inf _{\varphi \in C_{c}(X),\langle\varphi, \varphi\rangle=1} h(\varphi)=\max \{\lambda \in \mathbb{R} \mid(H-\lambda) \geq 0\}
$$

Proof. Let $m=1$. We close the form $h$ on $\ell^{2}(X)$ and consider the associated positive selfadjoint operator $H^{(1)}$. By the variational characterization of the bottom of the spectrum, the left hand side is equal to $\lambda_{0}\left(H^{(1)}\right)$. By Proposition 3.6 (a2) we have $D\left(H^{(1)}\right) \subseteq F(X)$. Thus, the asserted equality is an immediate consequence of Theorem 4.2 (b).

\section{Characterization of criticality}

In this section we discuss the notion of criticality by giving various characterizations of this notion. It turns out that being critical coincides with the notion of recurrence in the case $q=0$. 
In this subsection we define the notions of criticality, subcriticality and null-criticality that are fundamental for the present paper. In the continuum context these notions go back to B. Simon who coined the terms sub/super/critical-operators for Schrödinger operators with shortrange potentials which are defined on $\mathbb{R}^{d}, d \geq 3$ [35]. These notions were generalized by M. Murata [24] to Schrödinger operators which are defined in any subdomain of $\mathbb{R}^{d}$, where $d \geq 1$, and to general linear second-order elliptic operators with real coefficients by Y. Pinchover [25]. For more details see [27]. We remark that in the case of diffusion processes, transience (resp., positive-recurrence, null-recurrence) are the analogous notions to subcriticality (resp., positive-criticality, null-criticality) in the context of Schrödinger operators; we refer here to the monograph [31] (cf. Section 5).

Any function $w: X \rightarrow \mathbb{R}$ gives rise to a canonical quadratic form on $C_{c}(X)$ which we denote (with a slight abuse of notation) by $w$. It acts as

$$
w(\varphi):=\sum_{x \in X} w(x) \varphi(x)^{2} .
$$

Definition 5.1 (Critical/subcritical). Let $h$ be a quadratic form associated with a formal Schrödinger operator $H$ such that $h \geq 0$ on $C_{c}(X)$. The form $h$ is called subcritical in $X$ if there is a positive $w \in C(X)$ such that $h-w \geq 0$ on $C_{c}(X)$. Otherwise, the form $h$ is called critical in $X$.

The following characterization of criticality is well known in various contexts. For linear and quasilinear elliptic operators on $\mathbb{R}^{d}$, we refer to the following review papers [27, 28, 30, 31] and references therein. Within the theory of random walks and Dirichlet forms this phenomenon appears under the name recurrence, see e.g. [42, 8]. For positive matrices a corresponding phenomenon is called $r$-recurrence, [32, 40]. Furthermore, in the context of Riemannian manifolds such a notion appears under the name parabolicity. For the convenience of the reader we give a short and self-contained proof for our setting. In Remark 5.8 below we discuss how the result relates precisely to the ones in the other contexts that include the discrete setting.

\subsection{Criticality}

We need the following definition.

Definition 5.2 (Null-sequence). Let $h \geq 0$ on $C_{c}(X)$. A sequence $\left(e_{n}\right)$ in $C_{c}(X)$ of positive functions is called a null-sequence of $h$ if there exists $o \in X$ and $c>0$ such that $e_{n}(o)=c$ for all $n \geq 1$ and $h\left(e_{n}\right) \rightarrow 0$.

Theorem 5.3 (Criticality characterization). Let $b$ be a connected graph over $X$, let $q$ be a potential such that $h \geq 0$ on $C_{c}(X)$. Then, the following are equivalent:

(i) $h$ is critical in $X$.

(ii) $\lim _{\lambda \nearrow_{0}}\left(H^{(1)}-\lambda\right)^{-1} 1_{\{x\}}(y)=\infty$ for some (all) $x, y \in X$.

(ii') $\lim _{t \rightarrow \infty} \int_{0}^{t} \mathrm{e}^{-t H^{(1)}} 1_{\{x\}}(y) \mathrm{d} t=\infty$ for some (all) $x, y \in X$. 
(iii) There is a unique positive $H$-superharmonic function in $X$ (up to linear dependence).

(iii') There are only finitely many linearly independent positive $H$-superharmonic functions in $X$.

(iv) For any $o \in X$ and $c>0$, there is a null-sequence $\left(e_{n}\right)$ such that $e_{n}(o)=$ c for all $n \geq 0$.

(iv') There exists a positive $H$-harmonic function $v$ in $X$ and a null-sequence $\left(e_{n}\right)$ satisfying $0 \leq e_{n} \leq v$ and $e_{n}(x) \rightarrow v(x)$ for all $x \in X$.

(v) $\operatorname{cap}_{h}(\{x\}):=\inf \left\{h(\varphi) \mid \varphi \in C_{c}(X), \varphi(x)=1\right\}=0$ for all $x \in X$.

In particular, if one of the equivalent conditions holds true, then the unique (up to linear dependence) positive $H$-superharmonic function on $X$ is $H$-harmonic.

Definition 5.4 (Ground state). Let a critical form $h$ be given. For a given $o \in X$ we call the positive $H$-harmonic function $\psi$ with $\psi(o)=1$ given by the theorem above the (Agmon) ground state of $h$ normalized at $o$.

We extract some lemmas from the proof that we will use also in later parts of this paper.

Lemma 5.5. Let $h \geq 0$ on $C_{c}(X)$ be critical. Then there exists a non-trivial positive $H$ harmonic function and every positive $H$-superharmonic function is $H$-harmonic.

Proof. By part (a2) of Proposition 3.6, $D\left(H^{(1)}\right) \subseteq F(X)$. Hence, the Allegretto-Piepenbrink theorem implies that there exists a positive $H$-superharmonic function $v$. By the local Harnack inequality, Lemma 4.5, $v$ is strictly positive. Letting $w:=(H v) / v$, we observe that

$$
(H-w) v=0
$$

Hence, by the ground state transform, Proposition 4.8, we observe

$$
(h-w)(\varphi)=(h-w)_{v}(\varphi / v) \geq 0, \quad \varphi \in C_{c}(X),
$$

Hence, by criticality $(H v) / v=w \equiv 0$. Thus, $v$ is a positive $H$-harmonic function.

Lemma 5.6. Let $b$ be a connected graph over $X$, let $q$ be a potential and $w \geq 0$ be such that $h-w \geq 0$ on $C_{c}(X)$ and let $m$ be a measure of full support on $X$. Then

$$
w(x)\left(H^{(m)}-\lambda\right)^{-1} 1_{\{x\}}(x) \leq 1, \quad \lambda<0 \text { and } x \in X .
$$

Proof. We let $h^{(m)}$ be the closure of $h$ in $\ell^{2}(X, m)$. Then, for all $f \in D\left(h^{(m)}\right)$ with approximating sequence $\left(f_{n}\right)$ in $C_{c}(X)$ we have by Fatou's lemma

$$
h^{(m)}(f)=\lim _{n \rightarrow \infty} h^{(m)}\left(f_{n}\right) \geq \liminf _{n \rightarrow \infty}\left\langle w f_{n}, f_{n}\right\rangle_{m} \geq\langle w f, f\rangle_{m}
$$


Let $H^{(m)}$ be the positive self-adjoint operator associated to $h^{(m)}$. For $\lambda<0$ and a fixed $x \in X$, denote $g_{\lambda}:=\left(H^{(m)}-\lambda\right)^{-1} 1_{\{x\}}$. We find that for all $\lambda<0$

$$
\begin{aligned}
m(x) g_{\lambda}(x) & =\left\langle 1_{\{x\}}, g_{\lambda}\right\rangle_{m}=\left\langle\left(H^{(m)}-\lambda\right) g_{\lambda}, g_{\lambda}\right\rangle_{m} \\
& \geq h^{(m)}\left(g_{\lambda}\right) \geq\left\langle w g_{\lambda}, g_{\lambda}\right\rangle_{m} \geq m(x) w(x) g_{\lambda}(x)^{2} .
\end{aligned}
$$

Since $g_{\lambda}(x)>0$ (by Harnack's inequality), and as $m$ has full support, the statement follows.

Proof of Theorem 5.3. Let us first comment on the limit in (ii) and the equivalence of the formulation with "some" and "all": By the resolvent formula and positivity preservation of the resolvents (Corollary 3.5), the map $\lambda \mapsto\left(H^{(1)}-\lambda\right)^{-1} 1_{x}(y)$ is monotone increasing in $\lambda$ : precisely, for $\lambda \geq \mu$, one has

$$
\left(H^{(1)}-\lambda\right)^{-1} 1_{x}-\left(H^{(1)}-\mu\right)^{-1} 1_{x}=(\lambda-\mu)\left(H^{(1)}-\lambda\right)^{-1}\left(H^{(1)}-\mu\right)^{-1} 1_{x} \geq 0 .
$$

Hence, the limit either exists and is positive or it is infinite. Moreover, the functions $g_{\lambda}=$ $\left(H^{(1)}-\lambda\right)^{-1} 1_{x}$ are $H$-superharmonic (since $H^{(1)}$ is a restriction of $H$ by Corollary 3.8). Assuming the limit exists at one vertex $y$, these functions satisfy the assumptions of the Harnack principle, Lemma 4.6. Hence, the limits exist for all vertices, as $g_{\lambda}$ then converges to a $H$ superharmonic function. By the symmetry of $x, y$ in $\left(H^{(1)}-\lambda\right)^{-1} 1_{x}(y)$ due to self-adjointness of $H^{(1)}$ on $\ell^{2}(X)$ the limit then also exists for all $x$.

Let us now turn to show the equivalences.

(ii) $\Longleftrightarrow$ (ii'): This equivalence follows from Lemma 3.4 (the Laplace transform).

(iv) $\Longleftrightarrow(v)$ : This simply follows from the definition of cap c $_{h}$.

We proceed by proving that (i) $\Longleftrightarrow$ (iv).

(i) $\Longrightarrow$ (iv): Let $w_{n}=\frac{1}{n} 1_{\{o\}}$ for $o \in X$. Then the criticality implies for all $n \in \mathbb{N}$ the existence of $0 \leq e_{n} \in C_{c}(X)$ such that

$$
0 \leq h\left(e_{n}\right)<\sum_{x \in X} w_{n}(x) e_{n}(x)^{2}=\frac{1}{n} e_{n}(o)^{2} .
$$

In particular, $e_{n}(o)>0$ and, thus, $\left(e_{n}\right)$ can be chosen such that $e_{n}(o)=c$. Hence, $h\left(e_{n}\right) \leq \frac{c^{2}}{n}$ which implies that $\left(e_{n}\right)$ is a null-sequence.

(iv) $\Longrightarrow$ (i): Let $w \geq 0$ such that $h \geq w$ on $C_{c}(X)$. Choose $o \in X$ and $\left(e_{n}\right)$ as assumed. Then,

$$
0=\lim _{n \rightarrow \infty} h\left(e_{n}\right) \geq \limsup _{n \rightarrow \infty} \sum_{x \in X} w(x) e_{n}(x)^{2} \geq \limsup _{n \rightarrow \infty} w(o) e_{n}(o)^{2}=w(o) c^{2} .
$$

Since $c>0$, we infer $w(o)=0$. As $o$ can be chosen arbitrarily, we conclude $w \equiv 0$.

We show next that (i) \& (iv) $\Longrightarrow$ (iv') $\Longrightarrow$ (iii) $\Longrightarrow$ (iii') $\Longrightarrow$ (ii) $\Longrightarrow$ (i). 
(i) \& (iv) $\Longrightarrow$ (iv'): Let $v$ be a positive $H$-harmonic function whose existence is guaranteed by Lemma 5.5, Let $\left(e_{n}\right)$ be a null-sequence such that $e_{n}(o)=v(o)$, where $o \in X$. Let $h_{v}$ be the ground state transform with respect $v$. Then, since $h_{v}$ is Markovian

$$
h\left(e_{n} \wedge v\right)=h_{v}\left(v^{-1}\left(e_{n} \wedge v\right)\right)=h_{v}\left(v^{-1} e_{n} \wedge 1\right) \leq h_{v}\left(v^{-1} e_{n}\right)=h\left(e_{n}\right) \underset{n \rightarrow \infty}{\longrightarrow} 0 .
$$

Hence, we can replace $e_{n}$ by $\widetilde{e}_{n}:=e_{n} \wedge v$. Furthermore, $h_{v}\left(v^{-1} \widetilde{e}_{n}\right) \rightarrow 0$, thus, $\left(v^{-1} \widetilde{e}_{n}\right)$ converges pointwise to a constant and since $e_{n}(o)=v(o)$ have $v^{-1} \widetilde{e}_{n} \rightarrow 1$ pointwise as $n \rightarrow \infty$.

(iv') $\Longrightarrow$ (iii): Let $v$ be the positive $H$-harmonic function and $\left(e_{n}\right)$ be the null sequence such that $e_{n} \rightarrow v$ pointwise as $n \rightarrow \infty$ whose existence is assumed by (iv'). Let $u$ be a non-trivial positive $H$-superharmonic function which is strictly positive by the local Harnack inequality, Lemma 4.5. Then, by the ground state transform, we infer,

$$
0 \leq h_{u}\left(\frac{e_{n}}{u}\right) \leq h\left(e_{n}\right) \underset{n \rightarrow \infty}{\longrightarrow} 0 .
$$

Hence, there is a constant $c>0$ such that for all $x \in X$

$$
c=\lim _{n \rightarrow \infty} \frac{e_{n}(x)}{u(x)}=\frac{v(x)}{u(x)} .
$$

Thus, $v$ and $u$ are linearly dependent.

(iii) $\Longrightarrow$ (iii'): This is trivial.

(iii') $\Longrightarrow$ (ii): Suppose that the limit in (ii) is finite for all $x, y \in X$. Then for $x \in X$ the limit $G(x, \cdot)$ is a positive $H$-superharmonic function such that $H G(x, \cdot)=1_{\{x\}}$ by Lemma4.6. Hence, via $G(x, \cdot), x \in X$, there are infinitely many linearly independent positive $H$-superharmonic functions, and this contradicts (iii').

(ii) $\Longrightarrow$ (i): Assume that $h$ is subcritical. Then, there exists a positive $w$ such that $h-w \geq 0$ on $C_{c}(X)$. Then, for all $\lambda<0$ and a fixed $x \in \operatorname{supp} w$, we have by Lemma 5.6

$$
\lim _{\lambda \succ 0}\left(H^{(1)}-\lambda\right)^{-1} 1_{\{x\}}(x) \leq \frac{1}{w(x)}<\infty .
$$

Remark 5.7. Statement (iv') of Theorem 5.3 seems to be not known in the context of symmetric second-order elliptic differential operators, but indeed, it follows by the same argument as in the proof above.

There is a closely related phenomena called recurrence which is vastly studied in the literature. In the remark below we discuss how the results concerning recurrence for Dirichlet forms, random walks and positive matrices are related to our setting.

Remark 5.8. (a) First we look at random walks and positive matrices. Note that the operator $L$ in the decomposition of $H=L+q$ can be further decomposed into $L=D-A$ such that $D$ is the multiplication operator by the weighted vertex degree $d(x)=\sum_{y} b(x, y), x \in X$, and $A$ is the weighted adjacency matrix with entries $b(x, y), x, y \in X$. In random walks, one studies 
the transition matrix of a graph that is given by $P=D^{-1} A$. Then, recurrence is defined via the divergence of the sum $\sum_{n} P^{n}$ which can shown to be equivalent in the case $q=0$ to condition (ii) of Theorem 5.3, As we allow for non-vanishing $q$ our setting is more general. However, the setting of positive matrices, see e.g. [32, 40], includes the one of random walks and is much more flexible. There irreducible matrices with positive entries are studied. Given our setting of a connected graph, we observe that $h\left(1_{\{x\}}\right)=\operatorname{deg}(x)+q(x), x \in X$. Hence, $\operatorname{deg}+q \geq 0$ is implied by $h \geq 0$ and even $\operatorname{deg}+q>0$ since otherwise $1_{\{x\}}$ would be an eigenfunction and, therefore, $x$ is an isolated vertex. Hence, we can consider the matrix $(D+q)^{-1} A$, and this brings us into the realm of the theory of positive matrices. In this context the equivalence of (iv) and (v) and the existence of a unique positive solution have been proven in [32, 40].

(b) For Dirichlet forms the equivalences of the theorem are well known provided there is a suitable notion of superharmonic functions, see e.g. [8] except for (iii) and (iii'). This framework includes forms $h$ on graphs $b$ with potentials $q \geq 0$, see [17]. As we allow for non-positive $q$, our theory is a priori not included in the latter work. However, once one has shown the existence of a positive harmonic function the results can be carried over using the ground state transform. Unfortunately, this can be guaranteed a priori only for locally finite graphs, see [10].

\subsection{The extended space}

In this section we extend the form $h$ to a larger set of functions by taking an appropriate closure of $C_{c}(X)$. In the critical case we obtain an explicit representation of the action of $h$ on this closure. Furthermore, this allows us formulate an elegant negation of statement (iv) of the Theorem 5.3 to characterize subcriticality below.

Let $h \geq 0$ on $C_{c}(X)$ for a connected graph $b$ and a potential $q$ and fix $o \in X$. Define

$$
\|\varphi\|_{h, o}:=\sqrt{h(\varphi)+\varphi^{2}(o)}, \quad \varphi \in C_{c}(X)
$$

which is a priori a seminorm on $C_{c}(X)$. By the Green formula and the Harnack inequality we even have positive definiteness on $C_{c}(X)$, and we obtain a norm.

Lemma 5.9. Let $b$ be a connected graph over $X$, let $q$ be a potential such that $h \geq 0$ on $C_{c}(X)$.

(a) The norms $\|\cdot\|_{h, o}$ and $\|\cdot\|_{h, o^{\prime}}$ are equivalent, for any o and $o^{\prime}$ in $X$.

(b) Convergence with respect to $\|\cdot\|_{h, o}$ implies pointwise convergence.

Proof. Fix a positive $H$-supersolution $u$ on $X$. Let $o, o^{\prime} \in X$, and let a path $o=x_{0} \sim \ldots \sim$ $x_{n}=o^{\prime}$ be given. Set

$$
B_{o, o^{\prime}}:=\left(\sum_{i=0}^{n-1} \frac{1}{b\left(x_{i}, x_{i+1}\right) u\left(x_{i}\right) u\left(x_{i+1}\right)}\right) .
$$

Then, using a telescoping sum argument along the path and the Cauchy-Schwarz inequality yields by means of the ground state transform (see, Proposition 4.8),

$$
\left|\frac{\varphi(o)}{u(o)}-\frac{\varphi\left(o^{\prime}\right)}{u\left(o^{\prime}\right)}\right|^{2} \leq B_{o, o^{\prime}} h_{u}\left(\frac{\varphi}{u}\right) \leq B_{o, o^{\prime}} h(\varphi)
$$


for all $\varphi \in C_{c}(X)$. Thus, $\left|\frac{\varphi(o)}{u(o)}-\frac{\varphi\left(o^{\prime}\right)}{u\left(o^{\prime}\right)}\right|^{2}$ can be controlled by $h(\varphi)$, and therefore, the norms $\|\cdot\|_{h, o}$ and $\|\cdot\|_{h, o^{\prime}}$ are equivalent which is (a). Statement (b) follows directly from (a).

We denote by $D_{h, o}$ the completion of $C_{c}(X)$ with respect to $\|\cdot\|_{h, o}$. Note that in general such a space might not be a function space. But Lemma 5.9 implies that $D_{h, o}$ is a function space and hence a subspace of $C(X)$. So,

$$
D_{h, o}:={\overline{C_{c}(X)}}^{\|\cdot\|_{h, o}} \subset C(X) .
$$

In analogy to the theory of Dirichlet forms, we call it the extended space of the form $h$.

Note that by Lemma 5.9 above, $D_{h, o}$ does not depend on the choice of $o$ due to connectedness. Clearly, $h$ is closable on $D_{h, o}$ and we denote the closure by $\bar{h}$, i.e., for a sequence $\left(\varphi_{n}\right)$ in $C_{c}(X)$ that converges with respect to $\|\cdot\|_{h, o}$ to a function $\varphi \in D_{h, o}$ we let

$$
\bar{h}(\varphi):=\lim _{n \rightarrow \infty} h\left(\varphi_{n}\right) .
$$

By polarization $\bar{h}$ induces a bilinear form on $\bar{h}: D_{h, o} \times D_{h, o} \rightarrow \mathbb{R}$ and, hence, $\left(D_{h, o}, \bar{h}\right)$ becomes a semiscalar product space.

In the critical case there is an alternative way to define an extension of a form $h$ that satisfies $h \geq 0$ on $C_{c}(X)$. Let $v$ be the ground state of the critical form $h$ and let $h_{v}$ be its ground state transform. Note that since $h_{v}$ consists of a sum involving positive terms only, we can define $\widetilde{h}_{v}: C(X) \rightarrow[0, \infty]$ via

$$
\widetilde{h}_{v}(f):=\frac{1}{2} \sum_{x, y \in X} b(x, y) v(x) v(y)(f(x)-f(y))^{2}
$$

and

$$
\widetilde{D}_{h_{v}}=\left\{f \in C(X) \mid \widetilde{h}_{v}(f)<\infty\right\} .
$$

By Fatou's lemma $\widetilde{h}_{v}$ is lower semi-continuous and, thus, closed on $D_{h_{v}}$. This observation allows us to define $\widetilde{h}: C(X) \rightarrow[0, \infty]$ via

$$
\widetilde{h}(f):=\widetilde{h}_{v}\left(\frac{f}{v}\right)=\frac{1}{2} \sum_{x, y \in X} b(x, y) v(x) v(y)\left(\frac{f(x)}{v(x)}-\frac{f(y)}{v(y)}\right)^{2},
$$

and

$$
\widetilde{D}_{h}:=\{f \in C(X) \mid \widetilde{h}(f)<\infty\}=v^{-1} \widetilde{D}_{h_{v}} .
$$

The next theorem shows that in the critical case the forms $\bar{h}$ and $\widetilde{h}$ coincide.

Theorem 5.10. Let $b$ be a connected graph over $X$, let $q$ be a potential such that $h \geq 0$ on $C_{c}(X)$ is critical and $o \in X$. Then

$$
D_{h, o}=\widetilde{D}_{h}
$$

and for $f \in D_{h, o}$

$$
\bar{h}(f)=\widetilde{h}(f) .
$$


To prove this theorem we use some standard arguments from Dirichlet form theory. For the convenience of the reader we give the proofs.

Lemma 5.11. Let $q=0$, and $b$ be a graph such that $h \geq 0$ is critical. Let $\left(e_{n}\right)$ be a nullsequence for $h$, satisfying $0 \leq e_{n} \leq 1$. Then for any function $f$ we have

$$
\lim _{n \rightarrow \infty} h\left(e_{n} f\right)=\widetilde{h}(f)
$$

Proof. By Fatou's lemma we have

$$
h(f) \leq \liminf _{n \rightarrow \infty} h\left(f e_{n}\right)
$$

Thus, in order to show the reverse inequality it suffices to assume that $h(f)<\infty$. Assume first that $f$ is bounded. Using the elementary inequality

$$
(a b-c d)^{2}=[a(b-d)+d(a-c)]^{2} \leq 2 a^{2}(b-d)^{2}+2 d^{2}(a-c)^{2},
$$

for all $a, b, c, d \in \mathbb{R}$ and Tonelli's theorem to obtain

$$
\begin{aligned}
h\left(\left(1-e_{n}\right) f\right)= & \frac{1}{2} \sum_{x, y \in X} b(x, y)\left(\left(\left(1-e_{n}\right) f\right)(x)-\left(\left(1-e_{n}\right) f\right)(y)\right)^{2} \\
\leq & \sum_{x, y \in X} b(x, y)\left(1-e_{n}\right)^{2}(x)(f(x)-f(y))^{2} \\
& +\sum_{x, y \in X} b(x, y) f(y)^{2}\left(\left(1-e_{n}\right)(x)-\left(1-e_{n}\right)(y)\right)^{2} \\
\leq & \sum_{x, y \in X} b(x, y)\left(1-e_{n}\right)^{2}(x)(f(x)-f(y))^{2}+\|f\|_{\infty}^{2} h\left(e_{n}\right) .
\end{aligned}
$$

Letting $n \rightarrow \infty$, the right hand side tends to 0 . Indeed, the second term tends to 0 , since $0 \leq e_{n} \leq 1$ is a null-sequence, while the first term tends to 0 by the dominated convergence theorem since $h(f)<\infty$ (by the above assumption), and $e_{n} \rightarrow 1$ as $n \rightarrow \infty$, pointwise (use $F(x, y):=(f(x)-f(y))^{2}$ as a dominating function). On the other hand, by the reverse triangle inequality, we have

$$
\left|h\left(f e_{n}\right)^{1 / 2}-h(f)^{1 / 2}\right| \leq h\left(f\left(1-e_{n}\right)\right)^{1 / 2}
$$

and the statement follows for bounded $f$.

To obtain the statement for arbitrary $f$, note that

$$
\lim _{k \rightarrow \infty} \widetilde{h}(-k \vee f \wedge k)=\widetilde{h}(f)
$$

Indeed, the inequality " $\geq$ " follows by Fatou's lemma. The inequality " $\leq$ " follows since we have $\widetilde{h}_{v}\left(f_{k}\right) \leq \widetilde{h}_{v}(f)$, where $f_{k}:=-k \vee f \wedge k$ (which is the Markov property of $h(-k \vee f \wedge k) \leq$ $h(f))$. This finishes the proof. 
Proof of Theorem 5.10 Let $v$ be the ground state of $h$ and $h_{v}$ the ground state transform.

Let $f \in D_{h_{v}, o}$ and let $\left(f_{n}\right)$ be an approximating sequence in $C_{c}(X)$ with respect to $\|\cdot\|_{h_{v}, o}$. By the Lemma 5.9 we have pointwise convergence $f_{n} \rightarrow f$. Thus, by Fatou's lemma we have

$$
\widetilde{h}_{v}(f) \leq \lim _{n \rightarrow \infty} h_{v}\left(f_{n}\right)=\bar{h}_{v}(f)
$$

which in particular yields $D_{h_{v}, o} \subseteq \widetilde{D}_{h_{v}}$.

Since $h_{v}$ is critical (as $h$ is critical), let $\left(e_{n}\right)$ be a null-sequence converging to 1 pointwise (which exists according to Theorem 5.3 (iv')).

Let $f \in \widetilde{D}_{h_{v}}$. Then, since $\widetilde{h}_{v}(f)<\infty$, we can show $\widetilde{h}_{v}\left(f-e_{n} f\right) \rightarrow 0$ in the same way as in the proof of the previous Lemma 5.11: one starts with bounded $f$ and shows convergence to zero via dominated convergence; for general $f$, one uses approximations $f_{k}:=-k \vee f \wedge k$.

This yields $f \in D_{h_{v}, o}$ and by the lemma above and Fatou's lemma

$$
\bar{h}_{v}(f) \leq \lim _{n \rightarrow \infty} h_{v}\left(e_{n} f\right)=\widetilde{h}_{v}(f) .
$$

By what we have proven we deduce via the ground state transform

$$
D_{h, o}=v^{-1} D_{h_{v}, o}=v^{-1} \widetilde{D}_{h_{v}}=\widetilde{D}_{h},
$$

and for $f \in D_{h, o}$

$$
\widetilde{h}(f)=\widetilde{h}_{v}\left(\frac{f}{v}\right)=\bar{h}_{v}\left(\frac{f}{v}\right)=\bar{h}(f) .
$$

\subsection{Subcriticality}

In this section we reformulate Theorem 5.3 to give a characterization of subcriticality.

Theorem 5.12 (Subcriticality characterization). Let b be a connected graph over $X$, let $q$ be a potential such that $h \geq 0$ on $C_{c}(X)$. Then the following assertions are equivalent:

(i) $h$ is subcritical in $X$.

(ii) $0<\lim _{\lambda \nearrow 0}\left(H^{(1)}-\lambda\right)^{-1} 1_{\{x\}}(y)<\infty$ for some (all) $x, y \in X$.

(ii') $0<\int_{0}^{\infty} \mathrm{e}^{-t H^{(1)}} 1_{\{x\}}(y) \mathrm{d} t<\infty$ for some (all) $x, y \in X$.

(iii) For every vertex $x \in X$ there exists a positive $H$-superharmonic function $u$ such that $H u=1_{\{x\}}$ in $X$.

(iii') There are infinitely many linearly independent positive $H$-superharmonic functions in $X$.

(iv) The norms $\bar{h}(\cdot)^{1 / 2}$ and $\|\cdot\|_{h, o}$ are equivalent on $D_{h, o}$. In particular, $\left(D_{h, o}, \bar{h}\right)$ is a Hilbert space.

(iv') There is no positive $H$-harmonic function in $D_{h, o}$. 
(v) $\operatorname{cap}_{h}(\{x\})>0$ for all $x \in X$.

Proof. The equivalences (i) $\Longleftrightarrow$ (ii) $\Longleftrightarrow$ (ii') $\Longleftrightarrow$ (iii') $\Longleftrightarrow$ (iv) $\Longleftrightarrow$ (v) follow immediately from Theorem 5.3, where positivity in (ii) and (ii') is guaranteed by the Harnack principle (Lemma 4.6), and the Laplace transform (Lemma 3.4).

The implication (ii) $\Longrightarrow$ (iii) follows by Lemma 4.6 with a normalization at $x$. The implication (iii) $\Longrightarrow$ (iii') is clear.

(iv) $\Longrightarrow$ (iv'): Let $v$ be a non-negative $H$-harmonic function in $D_{h, o}$ and let $\left(v_{n}\right)$ be a sequence in $C_{c}(X)$ converging to $v$ with respect to $\|\cdot\|_{h, o}$. By Lemma 3.2, we can assume $\left|v_{n}\right| \leq v$, and therefore, by the virtue of Lebesgue's theorem $\lim _{n \rightarrow \infty} H v_{n}=H v=0$. So, for all $\varphi \in C_{c}(X)$, we conclude by the Green formula

$$
\bar{h}(v, \varphi)=\lim _{n \rightarrow \infty} h\left(v_{n}, \varphi\right)=\lim _{n \rightarrow \infty} \sum_{X} \varphi H v_{n}=0 .
$$

Since $\bar{h}$ is a scalar product by (iv) and $C_{c}(X)$ is dense in $D_{h, o}$, we infer $v=0$.

(iv') $\Longrightarrow$ (i): Assume $h$ is critical. Then, by Theorem 5.3 (iv') there is a positive harmonic function $v$ and a null sequence $\left(v_{n}\right)$ converging pointwise to $v$. Hence, $\left(v_{n}\right)$ is a $\|\cdot\|_{h, o}$ Cauchysequence in $D_{h, o}$. Lemma5.9 (b) and the pointwise convergence to $v$ implies that $v \in D_{h, o}$.

\subsection{The Green function}

In this section we take a closer look at the Green function which already occurred in Theorem 5.12 (iii). We show that it can be obtained also via an approximation of the Green function along an exhaustion of $X$. Fixing one argument, we show that the Green function is the minimal positive solution of the equation $H g=1_{x}$, and that it belongs to $D_{h, o}$.

Definition 5.13 (Green function). Let $b$ be a connected graph over $X$, let $q$ be a potential such that $h \geq 0$ on $C_{c}(X)$. If the integral

$$
G(x, y)=\int_{0}^{\infty} \mathrm{e}^{-t H^{(1)}} 1_{\{x\}}(y) \mathrm{d} t
$$

converges at a point $(x, y) \in X \times X, x \neq y$, (or equivalently, at all points $(x, y), x \neq y$ ), then $G$ is called the positive minimal Green function of $h$ in $X$.

Recall that by Theorem 5.12 (iii), the positive minimal Green function of $h$ exists in $X$ if and only if $h$ is subcritical in $X$. The minimality of $G$ is demonstrated in Theorem 5.16 (c).

Our aim is to show that $G$ can be obtained as the limit along an exhaustion. To this end we have to restrict $h$ and $H$ to finite sets and show that these restrictions are invertible. So, let $K \subseteq X$ be a finite subset and let $H_{K}$ be operator associated to the form $h$ restricted to $C_{c}(K)$. Note that the operators $H_{K}$ are restrictions of $H$.

We need the following minimum principle. 
Lemma 5.14 (Minimum principle). Let b be a connected graph over $X$, let $q$ be a potential such that $h \geq 0$ on $C_{c}(X)$. Let $K \subseteq X$ be a finite set and $u$ be $H$-superharmonic on $K$ and nonnegative outside $K$. Then, $u$ is nonnegative. Moreover, $u$ is either strictly positive in $K$ or $u=0$ in $K$.

Proof. Let $v$ be a strictly positive $H$-superharmonic function on $K$. Then, by Lemma 4.7, the function $u / v$ is $H_{v}$ superharmonic on $K$. For $H_{v}$ we can apply the minimum principle for operators on graphs with positive potentials, see e.g. the proof of [21, Theorem 8], and find that $u / v$ is nonnegative on $K$. Since $u$ was assumed to be nonnegative outside of $K$ as well, $u$ must be nonnegative, and by Harnack inequality $u$ is either strictly positive in $K$ or $u=0$ in $K$.

Lemma 5.15. Let $b$ be a connected graph over $X$, let $q$ be a potential such that $h \geq 0$ on $C_{c}(X)$ and let $K \subseteq X$ be a finite subset. Then, $H_{K}$ is invertible on $C_{c}(K)=\ell^{2}(K)$.

Proof. Let $v$ be a strictly positive $H$-superharmonic function given by Theorem 4.2 (Allegretto Piepenbrink-type theorem). Since $K$ is finite, $H_{K}$ not being invertible means there is $0 \neq u \in$ $C_{c}(K)$ such that $H_{K} u=0$. Assume $u \neq 0$ and $u$ is strictly positive at one point in $K$. Then $u$ is strictly positive on $K$ by the minimum principle, Lemma 5.14. Hence, by the ground state transform we have

$$
0=\left\langle u, H_{K} u\right\rangle=h(u) \geq h_{v}\left(\frac{u}{v}\right) \geq \sum_{x \in K, y \in X \backslash K} b(x, y) v(x) v(y) \frac{u(x)^{2}}{v(x)^{2}} .
$$

Since $v$ is strictly positive on $X$ and $u$ on $K$, we infer that $b(x, y)=0$ for all $x \in K$ and $y \in X \backslash K$. This is a contradiction to the connectedness of the graph.

With this preparation we can formulate the following statements about the Green function. Here, we call a sequence of finite subsets $\left(K_{n}\right)$ of $X$ an exhaustion of $X$ if $K_{n} \subseteq K_{n+1}, n \in \mathbb{N}$ and $\bigcup_{n} K_{n}=X$.

Theorem 5.16 (Properties of the Green function). Let b be a connected graph over $X$, let $q$ be a potential such that $h \geq 0$ on $C_{c}(X)$ and suppose that $h$ is subcritical in $X$.

(a) We have

$$
G(x, y)=\lim _{\lambda \nearrow 0}\left(H^{(1)}-\lambda\right)^{-1} 1_{\{x\}}(y)
$$

for all $x, y \in X$ and the convergence is monotone increasing. In particular, $G(x, y)=$ $G(y, x)$ for all $x, y \in X$, and $G(x, \cdot)$ and $G(\cdot, x)$ are superharmonic such that

$$
H G(x, \cdot)=H G(\cdot, x)=1_{\{x\}}, \quad x \in X .
$$

(b) We have

$$
G(x, y)=\lim _{n \rightarrow \infty} H_{K_{n}}^{-1} 1_{\{x\}}(y)
$$

for all $x, y \in X$ and all exhaustions $\left(K_{n}\right)$ of $X$ and the convergence is both pointwise monotone increasing and with respect to $\|\cdot\|_{h, o}$, i.e., for any $x \in X$, we have $G(x, \cdot) \in$ $D_{h, o}$ such that $G(x, \cdot)$ can be characterized in $D_{h, o}$ as follows: Let $g \in D_{h, o}$ and $x \in X$.

Then, the following statements are equivalent: 
(i) $g=G(x, \cdot)$.

(ii) $\bar{h}(g / g(x))=\inf \left\{h(f) \mid f \in C_{c}(X), f(x)=1\right\}$.

(iii) $H g=1_{x}$

(iv) $\bar{h}(g, f)=f(x)$ for all $f \in D_{h, o}$.

(c) $G(x, \cdot)$ is the smallest positive solution $g$ to the inequality

$$
H g \geq 1_{\{x\}}, \quad x \in X
$$

Proof of Theorem 5.16 (a) The first equality straightforwardly follows from the Laplace transform, Lemma 3.4. The monotonicity in the convergence follows from the resolvent identity

$$
\left(H^{(1)}-\lambda\right)^{-1} 1_{\{x\}}-\left(H^{(1)}-\mu\right)^{-1} 1_{\{x\}}=(\lambda-\mu)\left(H^{(1)}-\lambda\right)^{-1}\left(H^{(1)}-\mu\right)^{-1} 1_{\{x\}},
$$

and since by Corollary 3.5 , the resolvents are positivity preserving.

Letting $g_{\lambda, x}=\left(H^{(1)}-\lambda\right)^{-1} 1_{\{x\}}$, the symmetry follows since

$$
g_{\lambda, x}(y)=\left\langle\left(H^{(1)}-\lambda\right)^{-1} 1_{\{x\}}, 1_{\{y\}}\right\rangle=\left\langle 1_{x},\left(H^{(1)}-\lambda\right)^{-1} 1_{\{y\}}\right\rangle=g_{\lambda, y}(x) .
$$

The last statement of (a) follows from the Harnack principle (Lemma 4.6).

(b) Let $u_{n}=H_{K_{n}}^{-1} 1_{\{x\}}, n \in \mathbb{N}$. By the minimum principle (Lemma 5.14), we deduce $u_{n}-$ $u_{k} \geq 0$ on $K_{k}$ if $n \geq k$ and, hence, $\left(u_{n}\right)$ is monotone increasing. In addition, by the minimum principle (Lemma 5.14) applied to $u=G(x, \cdot)-u_{n}$, we have $0 \leq u_{n} \leq G(x, \cdot)$, on $K_{n}, n \in \mathbb{N}$. Therefore, the sequence $\left(u_{n}\right)$ has a limit $u$ that solves $H u=1_{\{x\}}$, by the Harnack principle (Lemma4.6). $0<u \leq G(x, \cdot)$.

Let $v$ be a strictly positive superharmonic function which exists by the Allegretto-Piepenbrink theorem (Theorem 4.2). Then by [17, Proposition 2.6 and 2.7], for the operator $H_{v}^{\left(v^{2}\right)}$ and its restrictions $H_{v, K}$ to $C_{c}(K)=\ell^{2}\left(K, v^{2}\right)$, we have for any $\lambda>0$

$$
\begin{array}{r}
T_{v}^{-1}\left(H_{K_{n}}-\lambda\right)^{-1} 1_{\{x\}}=\left(H_{v, K_{n}}-\lambda\right)^{-1} T_{v}^{-1} 1_{\{x\}} \\
\nearrow\left(H_{v}^{\left(v^{2}\right)}-\lambda\right)^{-1} T_{v}^{-1} 1_{\{x\}} \\
=T_{v}^{-1}\left(H^{(1)}-\lambda\right)^{-1} 1_{\{x\}}
\end{array}
$$

as $n \rightarrow \infty$, where the convergence is monotone increasing. Thus,

$$
G(x, \cdot)=\lim _{\lambda \nearrow 0}\left(H^{(1)}-\lambda\right)^{-1} 1_{\{x\}}=\lim _{\lambda \nearrow 0} \lim _{n \rightarrow \infty}\left(H_{K_{n}}^{(1)}-\lambda\right)^{-1} 1_{\{x\}}=\lim _{n \rightarrow \infty} H_{K_{n}}^{-1} 1_{\{x\}},
$$

where the limits interchange due to the monotone increasing limits in both parameters.

Moreover,

$$
h\left(g_{n}\right)=\left\langle H_{K_{n}} g_{n}, g_{n}\right\rangle=g_{n}(x) \leq G(x, x) \leq C .
$$

Hence, $\left(g_{n}\right)$ is a bounded sequence in the Hilbert space $\left(D_{h, o}, \bar{h}\right)$. By the Banach-Alaoglu theorem there exists a weakly convergent subsequence of $\left(g_{n}\right)$ whose limit in view of (b) coincides 
with the pointwise limit which is $g=G(x, \cdot)$. Hence, $G(x, \cdot) \in D_{h, o}$ and by Fatou's lemma $\bar{h}(G(x, \cdot)) \leq G(x, x)$. Hence by Green's formula

$$
\bar{h}\left(G(x, \cdot)-g_{n}\right)=\bar{h}(G(x, \cdot))-2 \sum_{y \in X} H G(x, y) g_{n}(y)+\sum_{y \in X} H g_{n}(y) g_{n}(y) \leq G(x, x)-g_{n}(x)
$$

which converges to 0 as $n \rightarrow \infty$. Hence, $g_{n}$ converges to $G(x, \cdot)$ with respect to $\|\cdot\|_{h, o}$. Moreover, this as well immediately yields

$$
\bar{h}(G(x, \cdot))=G(x, x) .
$$

Let us turn to the equivalence.

(i) $\Longrightarrow$ (ii): It is elementary to see that $g_{n}=H_{K_{n}}^{-1} 1_{x} / H_{K_{n}}^{-1} 1_{x}(x), n \in \mathbb{N}$, minimizes the restriction $h_{n}$ of $h$ to $\left\{\varphi \in C_{c}\left(K_{n}\right) \mid \varphi(x)=1\right\}$ for an exhaustion $\left(K_{n}\right)$ of $X$ with finite sets and $x \in K_{n}$. Therefore, we have for all $f \in C_{c}(X)$ with $f(x)=1$

$$
\bar{h}(G(x, \cdot) / G(x, x))=\lim _{n \rightarrow \infty} h_{n}\left(g_{n}\right) \leq \lim _{n \rightarrow \infty} h_{n}(f)=\lim _{n \rightarrow \infty} h(f)
$$

(ii) $\Longrightarrow$ (iii): Assume $\bar{g}=g / g(x)$ minimizes $\bar{h}$ on $\left\{f \in D_{h, o} \mid f(x)=1\right\}$. Hence, the function $t \mapsto \bar{h}\left(\bar{g}+t 1_{y}\right)$ has derivative zero at $t=0$ for all $y \neq x$, i.e., we have

$$
0=\left.\frac{d}{d t} \bar{h}\left(\bar{g}+t 1_{y}\right)\right|_{t=0}=2 \bar{h}\left(\bar{g}, 1_{y}\right)=2 H \bar{g}(y) .
$$

So, does the function $t \mapsto \bar{h}\left((1-t) \bar{g}+t 1_{x}\right)$, i.e.,

$$
0=\left.\frac{d}{d t} \bar{h}\left((1-t) \bar{g}+t 1_{x}\right)\right|_{t=0}=-2 \bar{h}(\bar{g})+2 \bar{h}\left(\bar{g}, 1_{x}\right)=2(1-H g(x)) .
$$

This proves $H g=1_{x}$.

(iii) $\Longrightarrow$ (iv): This follows directly by Green's formula for all $f \in C_{c}(X)$ and by density for all functions in $D_{h, o}$.

(iv) $\Longrightarrow$ (i): By Theorem5.12(iv), $\left(D_{h, o}, \bar{h}\right)$ is a Hilbert space. Moreover, by Lemma5.9(b) the map $\delta_{x}: D_{h, o} \rightarrow \mathbb{R}, f \mapsto f(x)$ is a continuous linear functional. Hence, by the Riesz representation theorem there is a unique $g \in D_{h, o}$ such that $\bar{h}(g, f)=\delta_{x}(f)=f(x)$. However, by Green's formula $G(x, \cdot)$ satisfies the equation $\bar{h}(G(x, \cdot), \varphi)=\varphi(x)$ for all $\varphi \in C_{c}(X)$. By density of $C_{c}(X)$ in $D_{h, o}$ we infer $g=G(x, \cdot)$.

(c) Let $u$ be a $H$-superharmonic function such that $H u \geq 1_{\{x\}}$ and $g_{n}=H_{K_{n}}^{-1} 1_{\{x\}}$ for an exhaustion $\left(K_{n}\right)$. Then, by the minimum principle we infer $u-g_{n} \geq 0$ on $K_{n}$. By (b) we infer $u \geq G(x, \cdot)$.

\subsection{Uniform subcriticality}

In this section we turn to the topic of uniform subcriticality. In the continuum this notion goes back to work of Pinchover [25]. In the discrete setting of random walks this notion is known as 
uniform transience and was investigated in the joint work of Barlow, Coulhon, and Grigor'yan [1], and in the paper of Windisch [41], and also in the works of Kasue [13, 14] under no particular name. There is also a recent work which discusses the Dirichlet problem for the Royden boundary on uniformly transient graphs [18].

Definition 5.17 (Uniform subcriticality). Let $b$ be a connected graph over $X$, let $q$ be a potential such that $h \geq 0$ on $C_{c}(X)$. We say $h$ is uniformly subcritical if there is a constant $C>0$ such that for every $x \in X$ there is $w \geq 0$ such that $w(x) \geq C$ and $h-w \geq 0$ on $C_{c}(X)$.

We denote

$$
C_{0}(X):={\overline{C_{c}}}^{\|\cdot\|_{\infty}}
$$

The following characterization is shown in the continuum setting [25]. For Laplace type operators on graphs (with non-negative potentials) a similar results are found in [13, 14, 18].

Theorem 5.18 (Uniform subcriticality characterization). Let b be a connected graph over $X$, let $o \in X$, let $q$ be a potential such that $h \geq 0$ on $C_{c}(X)$. Then the following assertions are equivalent:

(i) $h$ is uniformly subcritical in $X$.

(i’) There is $C>0$ such that

$$
h(\varphi) \geq C\|\varphi\|_{\infty}^{2}, \quad \varphi \in C_{c}(X)
$$

(i”) There is $C>0$ such that

$$
\|\varphi\|_{h, o} \geq C\|\varphi\|_{\infty}^{2}, \quad \varphi \in C_{c}(X)
$$

(ii) There is $C>0$ such that $G(x, x) \leq C$ for all $x \in X$.

(iii) There is $C>0$ such that for every vertex $x \in X$, there exists a positive $H$-superharmonic function $u(x) \leq C$ such that $H u \geq 1_{\{x\}}$ in $X$.

(iv) $D_{h, o} \subseteq C_{0}(X)$.

(v) There is $C>0$ such that $\inf _{x \in X} \operatorname{cap}_{h}(\{x\})>C$ for all $x \in X$.

In particular, $G(x, \cdot) \in C_{0}(X)$ for all $x \in X$.

Proof. The equivalences (i) $\Longleftrightarrow$ (i') $\Longleftrightarrow$ (v) follow directly from the definitions.

The implications (i') $\Longrightarrow$ (i’) $\Longrightarrow$ (iv) are clear.

(iv) $\Longrightarrow$ (i'): By the closed graph theorem the map $\left(D_{h, o},\|\cdot\|_{o}\right) \rightarrow\left(C_{0}(X),\|\cdot\|_{\infty}\right)$ is continuous for any $o \in X$. Hence, (i”) follows. 
(i) $\Longrightarrow$ (ii): This follows from Lemma 5.6,

(ii) $\Longrightarrow$ (i): Let $g=G(x, \cdot), x \in X$. Then, by the ground state transform, we have with $w=1 / g(x)$

$$
h(\varphi)=h_{g}\left(\frac{\varphi}{g}\right)+\langle w \varphi, \varphi\rangle \geq\langle w \varphi, \varphi\rangle
$$

Since $G(x, x)<C, x \in X$, we have $w(x) \geq 1 / C$ and, therefore, $h$ is uniformly subcritical.

(iv) $\Longrightarrow$ (iii): This follows from Theorem 5.16 (d).

(iii) $\Longrightarrow$ (ii): This follows from Theorem 5.16(c).

The "in particular" follows by (iv) combined with Theorem 5.16 (d).

\section{Characterization of positive/null-criticality}

Below, we provide a characterization of null/positive-criticality which is defined next.

Definition 6.1 (Null-critical/positive-critical). Let $h$ be a quadratic form associated with the formal Schrödinger operator $H$, such that $h \geq 0$ on $C_{c}(X)$. The form $h$ is called null-critical (resp., positive-critical) in $X$ with respect to a positive potential $w$ if $h$ is critical in $X$ and $\sum_{X} \psi^{2} w=\infty$ (resp., $\left.\sum_{X} \psi^{2} w<\infty\right)$, where $\psi$ is the ground state of $h$ in $X$.

Note that the null/positive-criticality of a critical form depends also on the weight $w$.

For any nontrivial positive function $w$ consider the seminorm $\|\cdot\|_{w}$ by

$$
\|f\|_{w}=\left(\sum_{x \in X} w(x) f(x)^{2}\right)^{1 / 2}
$$

Whenever, $w$ is strictly positive, then $\|\cdot\|_{w}$ is a norm. We can close the form $h$ in $\ell^{2}(X, w)$ (cf. Theorem 3.1), and associate a self-adjoint operator $H^{(w)}$ to the closure.

We prove the statement for the form $h-w$ instead of $h$ because this is how it is often used. Hence we use the form $h-w \geq 0$ to define the operator $(H-w)^{(w)}$. Recall that by Theorem 5.3, a critical operator admits a unique ground state (up to normalization).

Theorem 6.2 (Positive criticality). Let $b$ be a connected graph over $X$, and let $q$ be a potential and $o \in X$. Let $w \supsetneqq 0$ such that $h-w \geq 0$ is critical in $X$, and let $\psi$ be the corresponding ground state of $h-w$ normalized at o. Then, the following statements are equivalent:

(i) $h-w$ is positive-critical with respect to $w$. That is $\psi \in \ell^{2}(X, w)$.

(ii) $\psi \in D_{h, o}$.

(iii) $\psi \in D_{h, o} \cap \ell^{2}(X, w)$. 
(iv) There is $v \in D_{h, o} \cap \ell^{2}(X, w)$ such that

$$
\frac{\bar{h}(v)}{\|v\|_{w}^{2}}=\inf _{\varphi \in C_{c}(X)} \frac{h(\varphi)}{\|\varphi\|_{w}^{2}} .
$$

(v) $\psi \in D_{h, o} \cap \ell^{2}(X, w)$ and

$$
\frac{\bar{h}(\psi)}{\|\psi\|_{w}^{2}}=\inf _{\varphi \in C_{c}(X)} \frac{h(\varphi)}{\|\varphi\|_{w}^{2}}
$$

Furthermore, if $w$ is strictly positive, then also the following statement is equivalent:

(vi) $\psi$ is an eigenfunction of the operator $(H-w)^{(w)}$ with eigenvalue 0.

Proof. Since $h-w$ is critical, it follows that $h \geq 0$, and we have

$$
\inf _{\varphi \in C_{c}(X)} \frac{h(\varphi)}{\|\varphi\|_{w}^{2}}=1 .
$$

Furthermore, for any null-sequence $\left(\varphi_{n}\right)$ in $C_{c}(X)$ with $\varphi_{n}(o)=\psi(o)$ and $\lim _{n \rightarrow \infty}(h-$ $w)\left(\varphi_{n}\right)=0$, we have by Theorem 5.3 (iv'), $\varphi_{n} \rightarrow \psi$ pointwise. This gives the equivalence "(i) $\Longleftrightarrow$ (ii) $\Longleftrightarrow$ (iii) $\Longleftrightarrow$ (iv) $\Longleftrightarrow$ (v)" with $v=\psi$ in (iv).

Now, assume that $w$ is strictly positive. Then (vi) $\Longrightarrow$ (i) is obvious because $\psi$ being an eigenfunction implies $\psi \in \ell^{2}(X, w)$.

We finally prove (iii) $\Longrightarrow$ (vi). In order to show $\psi \in D\left((H-w)^{(w)}\right)$ it suffices to demonstrate $\psi \in D\left((h-w)^{(w)}\right)$. Then $\psi \in D\left((H-w)^{(w)}\right)$ follows directly from the definition of the operator domain using that $C_{c}(X)$ is dense in the form domain, $(H-w) \psi=0$ and the Green formula (Lemma 2.1). Since the ground state transform $(h-w)_{\psi}$ is Markovian we have by general Dirichlet form theory

$$
D\left((h-w)_{\psi}^{(m)}\right)=D_{(h-w)_{\psi}, o} \cap \ell^{2}(X, m)
$$

for any measure $m$ and $o \in X$, (see [8, Theorem 1.5.2] or for the special case of graphs [18, Lemma 1.6]). Hence, by the ground state transform we get

$$
\begin{aligned}
D\left((h-w)^{(w)}\right) & =T_{\psi^{-1}} D\left((h-w)_{\psi}^{\left(\psi^{2} w\right)}\right) \\
& =T_{\psi^{-1}}\left(D_{(h-w)_{\psi}, o} \cap \ell^{2}\left(X, \psi^{2} w\right)\right) \\
& =D_{h-w, o} \cap \ell^{2}(X, w) .
\end{aligned}
$$

This shows the claim.

Positive criticality with respect to the weight $w=1$ is characterized also by the large time behavior of the heat kernel. For the counterpart result in the case of second-order elliptic partial differential operators, see [28] and references therein. 
Theorem 6.3 ([19, Theorem 3.1 and Corollary 5.6]). Let $H$ be a Schrödinger operator on $X$, and suppose that $H \geq 0$ in $X$. Let $\lambda_{0}:=\lambda_{0}\left(H^{(1)}\right)$ be the bottom of the spectrum of the selfadjoint operator $H^{(1)}$, and denote by $p_{t}(x, y):=\mathrm{e}^{-t H^{(1)}} 1_{\{x\}}(y)$ the heat kernel of operator $H$. Then for each $x, y \in X$

$$
\lim _{t \rightarrow \infty} \frac{\log p_{t}(x, y)}{t}=-\lambda_{0}
$$

Moreover,

$$
\lim _{t \rightarrow \infty} \mathrm{e}^{\lambda_{0} t} p_{t}(x, y)=\Psi(x) \Psi(y),
$$

where $\Psi=0$ unless $H-\lambda_{0}$ is positive critical in $X$, and in this case, $\Psi \in \ell^{2}(X)$ is the normalized ground state of $H-\lambda_{0}$.

Let $H \geq 0$ in $X$. The following theorem demonstrates that positive criticality with respect to a weight $w$ is characterized by the behavior of the positive minimal Green function of the operator $H-\lambda w$ as $\lambda \nearrow 0$. For the counterpart result in the case of second-order elliptic partial differential operators, see [26, Theorem 1.1].

Theorem 6.4. Let $H$ be a Schrödinger operator on $X$, and suppose that $H \geq 0$ in $X$. Let $w$ be a positive weight on $X$. For $\lambda<0$, let $G_{\lambda}\left(x, x_{0}\right)$ be the positive minimal Green function of the operator $H-\lambda w$ on $X$. Then for each $x, x_{0} \in X$ we have

$$
\lim _{\lambda \nearrow 0}(-\lambda) G_{\lambda}\left(x, x_{0}\right)=\phi(x) \phi\left(x_{0}\right)
$$

where $\phi=0$ unless $H$ is positive critical in $X$ with respect to the weight $w$, and in this case, $\phi$ is the normalized ground state of $H$ in $\ell^{2}(X, w)$.

Proof. If $H$ subcritical, then clearly

$$
\lim _{\lambda \nearrow 0}(-\lambda) G_{\lambda}\left(x, x_{0}\right)=0
$$

So, we may assume that $H$ is critical in $X$.

Fix $x_{0}, x_{1} \in X$. For $s<0$, let $G_{s}\left(\cdot, x_{0}\right)$ be the minimal positive Green function of $H-s W$ in $X$ satisfying $(H-s w) G\left(\cdot, x_{0}\right)=\delta_{x_{0}}$.

Set

$$
u_{s}(x):=\frac{G_{s}\left(x, x_{0}\right)}{G_{s}\left(x_{1}, x_{0}\right)} .
$$

Claim: For any $x \in X$

$$
\lim _{s \nearrow 0} u_{s}(x)=\frac{\phi(x)}{\phi\left(x_{1}\right)},
$$

where $\phi$ is the unique positive ground state for $H$.

Indeed, for any fixed $t<s<0$, the function $u_{s}$ satisfies

$$
(H-t w) u_{s} \geq 0 \quad \text { in } X,
$$


as well as $u_{s}\left(x_{1}\right)=1$. Therefore, by the Harnack principle, a standard exhaustion and diagonalization arguments, and up to a subsequence, the sequence $\left(u_{s}\right)$ converges to a positive supersolution $\varphi$ the equation $(H-t w) u=0$ in $X$.

Letting $t \nearrow 0$, we obtain by Fatou's Lemma that pointwise, $H \varphi \geq 0$ in $X$. So, $\varphi$ is a positive super solution of the equation $H u=0$ in $X$ satisfying $\varphi\left(x_{1}\right)=1$. The criticality of $H$ in $X$ implies now that $\varphi(x)=\phi(x) / \phi\left(x_{1}\right)$. In particular, the limit does not depend on the subsequence, hence, $\lim _{s} \nearrow_{0} u_{s}(x)=\phi(x) / \phi\left(x_{1}\right)$, and the claim is proved.

Now, by the resolvent equation and the symmetry of the Green's function, we have for any $t<s<0$

$$
G_{s}\left(x, x_{0}\right)=G_{t}\left(x, x_{0}\right)+(s-t) \sum_{z \in X} w(z) G_{t}(x, z) G_{s}\left(z, x_{0}\right)
$$

Consequently,

$$
\frac{G_{s}\left(x, x_{0}\right)}{G_{s}\left(x_{1}, x_{0}\right)}=\frac{G_{t}\left(x, x_{0}\right)}{G_{s}\left(x_{1}, x_{0}\right)}+(s-t) \sum_{z \in X} w(z) G_{t}(x, z) \frac{G_{s}\left(z, x_{0}\right)}{G_{s}\left(x_{1}, x_{0}\right)} .
$$

Hence,

$$
\frac{G_{s}\left(x, x_{0}\right)}{G_{s}\left(x_{1}, x_{0}\right)} \geq(s-t) \sum_{z \in X} w(z) G_{t}(x, z) \frac{G_{s}\left(z, x_{0}\right)}{G_{s}\left(x_{1}, x_{0}\right)} .
$$

Let $s \nearrow 0$. By the claim proven above and using Fatou's lemma, this yields

$$
\frac{\phi(x)}{\phi\left(x_{1}\right)} \geq-t \sum_{z \in X} w(z) G_{t}(x, z) \frac{\phi(z)}{\phi\left(x_{1}\right)} .
$$

Fix $t<0$ and let

$$
v_{t}(x):=-t \sum_{z \in X} w(z) G_{t}(x, z) \phi(z)
$$

Then

$$
H v_{t}=-t w\left(\phi-v_{t}\right)
$$

By (6.4) we have that $H v_{t} \geq 0$ in $X$. Moreover, (6.4) implies that $v_{t}$ is a positive supersolution of the equation $H u=0$ in $X$. By the maximum principle, either $v_{t}$ is strictly positive or identically zero. So, $v_{t}>0$. But, due the criticality of $H$ in $X$, it follows that $v_{t}$ is a solution, and $\phi-v_{t}$ are either strictly positive solution of the equation $H u=0$ in $X$, or the zero solution. Consequently, $v_{t}=\alpha \phi$, where $0<\alpha \leq 1$. In fact, $\alpha=1$ since otherwise, (6.5) implies the $v_{t}$ is strictly positive supersolution. So,

$$
\phi(x)=-t \sum_{z \in X} w(z) G_{t}(x, z) \phi(z) \quad \forall x \in X .
$$

Taking the liminf as $t \nearrow 0$ on the right hand side of (6.6), an easy computation involving again Fatou's lemma, shows that one obtains a positive supersolution of the equation $H u=0$ in $X$. Consequently, by uniqueness of those supersolutions, we arrive at

$$
\phi(x)=\lim _{t \nearrow 0}\left(-t \sum_{z \in X} G_{t}(x, z) w(z) \phi(z)\right) .
$$


Hence,

$$
\phi(x)=\lim _{t \nearrow 0}\left(-t G_{t}\left(x, x_{0}\right) \sum_{z \in X} \frac{G_{t}(x, z)}{G_{t}\left(x, x_{0}\right)} w(z) \phi(z)\right),
$$

which in turn gives

$$
\limsup _{t \nearrow 0}\left(-t G_{t}\left(x, x_{0}\right)\right)=\underset{t \nearrow 0}{\limsup }\left(\frac{\phi(x)}{\sum_{z} \frac{G_{t}(x, z)}{G_{t}\left(x, x_{0}\right)} w(z) \phi(z)}\right) .
$$

Therefore, Fatou's lemma yields

$$
\limsup _{t \nearrow 0}\left(-t G_{t}\left(x, x_{0}\right)\right) \leq \frac{\phi(x) \phi\left(x_{0}\right)}{\sum_{z} \phi^{2}(z) w(z)}
$$

In particular, if $H$ is null-critical with respect to $w$, then $\lim \sup _{t \succ 0}\left(-t G_{t}\left(x, x_{0}\right)\right)=0$.

It remains to deal with the positive-critical case.

Note that by (6.8), the sequence $\left(-t G_{t}\left(x, x_{0}\right)\right)$ is locally bounded, and therefore, as above, and up to a subsequence, it converges to a positive supersolution of the equation $H u=0$ in $X$. Recall that the ground state $\phi$ is the unique supersolution of the equation $H u=0$ in $X$. Consequently, for any subsequence $t_{j} \nearrow 0$ there exists $\beta \geq 0$ such that

$$
\lim _{t_{j} \nearrow 0}\left(-t_{j} G_{t_{j}}\left(x, x_{0}\right)\right)=\beta \frac{\phi(x) \phi\left(x_{0}\right)}{\sum_{z} \phi^{2}(z) w(z)} .
$$

We observe from inequality (6.8) that $\beta \leq 1$. We still need to show that $\beta \geq 1$. For this purpose, note first that the inequality (6.8) holds true for all $x_{0}=z \in X$. Combining this with inequality (6.7) and with Fatou's lemma, one shows that

$$
\phi\left(x_{1}\right) \leq \sum_{z \in X} \lim _{j \rightarrow \infty}\left(-t_{j}\right) G_{t_{j}}\left(x_{1}, z\right) w(z) \phi(z) \leq \beta \frac{\phi\left(x_{1}\right) \sum_{z} \phi^{2}(z) w(z)}{\sum_{z} \phi^{2}(z) w(z)} .
$$

So, $\beta \geq 1$. Since the subsequence $\left(t_{j}\right)$ was chosen arbitrarily, the proof of the theorem is finished.

Remark 6.5. Using (6.3) and the Martin boundary approach it follows that for any positive potential $w$, inequality (6.4) holds even if $H$ is subcritical in $X$ and $\phi$ is any positive solution of the equation $H u=0$ in $X$. Moreover, as it is shown above, in the critical case equality holds true for any $x \in X$ (see (6.6)).

Remark 6.6. For the weight $w=1$, Theorem 6.4 follows directly from Theorem 6.3 using a classical Abelian theorem [34, Theorem 10.2].

Acknowledgments. M. K. is grateful to the Department of Mathematics at the Technion for the hospitality during his visits and acknowledges the financial support of the German Science Foundation. Y. P. and F. P. acknowledge the support of the Israel Science Foundation (grants No. 970/15) founded by the Israel Academy of Sciences and Humanities. F. P. is grateful for support through a Technion Fine Fellowship. 


\section{References}

[1] M. Barlow, T. Coulhon and A. Grigor'yan, Manifolds and graphs with slow heat kernel decay. Invent. Math., 144 (2001), 609-649.

[2] S. Beckus and Y. Pinchover, Shnol-type theorem for the Agmon ground state. arXiv: 1706.04869.

[3] M. Bonnefont and S. Golénia, Essential spectrum and Weyl asymptotics for discrete Laplacians. Ann. Fac. Sci. Toulouse Math. (6), 24 (2015), 563-624.

[4] J. Dodziuk, Difference equations, isoperimetric inequality and transience of certain random walks. Trans. Amer. Math. Soc., 284(1984), 787-794.

[5] R. L. Frank, D. Lenz and D. Wingert, Intrinsic metrics for non-local symmetric Dirichlet forms and applications to spectral theory. J. Funct. Anal., 266 (2014), 4765-4808.

[6] R. L. Frank and R. Seiringer, Non-linear ground state representations and sharp Hardy inequalities. J. Funct. Anal., 255 (2008), 3407-3430.

[7] R. L. Frank, B. Simon and T. Weidl, Eigenvalue bounds for perturbations of Schrödinger operators and Jacobi matrices with regular ground states. Comm. Math. Phys., 282 (2008), 199-208.

[8] M. Fukushima, Y. Oshima and M. Takeda, Dirichlet Forms and Symmetric Markov Processes. de Gruyter Studies in Mathematics 19, extended edition, Walter de Gruyter \& Co., Berlin 2011.

[9] B. Güneysu, M. Keller and M. Schmidt, A Feynman-Kac-Itô formula for magnetic Schrödinger operators on graphs. Probab. Theory Related Fields, 165 (2016), 365-399.

[10] S. Haeseler and M. Keller, Generalized solutions and spectrum for Dirichlet forms on graphs. In Random walks, boundaries and spectra, Progr. Probab. 64, Birkhäuser/Springer Basel AG, Basel, 2011, 181-199.

[11] S. Haeseler, M. Keller, D. Lenz and R. Wojciechowski, Laplacians on infinite graphs: Dirichlet and Neumann boundary conditions. J. Spectr. Theory, 2 (2012), 397-432.

[12] S. Haeseler, M. Keller, and R. K. Wojciechowski, Volume growth and bounds for the essential spectrum for Dirichlet forms. J. Lond. Math. Soc. (2), 88 (2013), 883-898.

[13] A. Kasue, Convergence of metric graphs and energy forms. Rev. Mat. Iberoam., 26 (2010), 367-448.

[14] A. Kasue, Random walks and Kuramochi boundaries of infinite networks. Osaka J. Math., 50 (2013), 31-51.

[15] M. Keller, Applications of Operator Theory - Discrete Operators. Lecture notes, 2012.

[16] M. Keller and D. Lenz, Unbounded Laplacians on graphs: basic spectral properties and the heat equation. Math. Model. Nat. Phenom., 5 (2010), 198-224.

[17] M. Keller and D. Lenz, Dirichlet forms and stochastic completeness of graphs and subgraphs. J. Reine Angew. Math., 666 (2012), 189-223.

[18] M. Keller, D. Lenz, M. Schmidt and R. K. Wojciechowski, Note on uniformly transient graphs. to appear in Rev. Mat. Iberoam., 2017.

[19] M. Keller, D. Lenz, H. Vogt and R. Wojciechowski, Note on basic features of large time behaviour of heat kernels. J. Reine Angew. Math., 708 (2015), 73-95.

[20] M. Keller, Y. Pinchover and F. Pogorzelski, Optimal Hardy inequalities for Schrödinger operators on graphs. arXiv: 1612.04051.

[21] H. Kovařík and A. Laptev, Hardy inequalities for Robin Laplacians. J. Funct. Anal., 262 (2012), 4972-4985.

[22] D. Lenz, and P. Stollmann, Peter and I. Veselić, The Allegretto-Piepenbrink theorem for strongly local Dirichlet forms Doc. Math. 14 (2009), 167-189.

[23] B. Mohar and W. Woess, A survey on spectra of infinite graphs. Bull. London Math. Soc., 21 (1989), 209-234.

[24] M. Murata, Structure of positive solutions to $(-\Delta+V) u=0$ in $\mathbf{R}^{n}$. Duke Math. J., 53 (1986), 869-943.

[25] Y. Pinchover, On positive solutions of second-order elliptic equations, stability results, and classification. Duke Math. J., 57 (1988), 955-980. 
[26] Yehuda Pinchover. Large time behavior of the heat kernel and the behavior of the Green function near criticality for nonsymmetric elliptic operators. J. Funct. Anal., 104(1):54-70, 1992.

[27] Y. Pinchover, Topics in the theory of positive solutions of second-order elliptic and parabolic partial differential equations. In Spectral theory and mathematical physics: a Festschrift in honor of Barry Simon's 60th birthday, Proc. Sympos. Pure Math. 76, Amer. Math. Soc., Providence, RI, 2007, 329-355.

[28] Y. Pinchover, Some aspects of large time behavior of the heat kernel: an overview with perspectives. In Mathematical physics, spectral theory and stochastic analysis, Oper. Theory Adv. Appl. 232, Birkhäuser/Springer Basel AG, Basel, 2013, 299-339.

[29] Y. Pinchover and G. Psaradakis, On positive solutions of the $(p, A)$-Laplacian with a potential in morrey space. Anal. PDE 9 (2016), 1317-1358.

[30] Y. Pinchover and K. Tintarev, On positive solutions of $p$-Laplacian-type equations. In Analysis, partial differential equations and applications, Oper. Theory Adv. Appl. 193, Birkhäuser Verlag, Basel, 2009, 245 267.

[31] R. G. Pinsky, Positive Harmonic Functions and Diffusion. Cambridge Studies in Advanced Mathematics 45, Cambridge University Press, Cambridge 1995.

[32] W. E. Pruitt, Eigenvalues of non-negative matrices. Ann. Math. Statist. 35 (1964), 1797-1800.

[33] M. Reed and B. Simon, Methods of Modern Mathematical Physics. IV. Analysis of Operators. Academic Press [Harcourt Brace Jovanovich, Publishers], New York-London, 1978.

[34] B. Simon, Functional Integration and Quantum Physics, Pure and Applied Mathematics, 86, Academic Press, Inc., New York-London, 1979.

[35] B. Simon, Brownian motion, $L^{p}$ properties of Schrödinger operators and the localization of binding. $J$. Funct. Anal. 35 (1980), 215-229.

[36] M. Takeda, Criticality and subcriticality of generalized Schrödinger forms. Illinois J. Math. 58 (2014), 251-277.

[37] M. Takeda, Criticality for Schrödinger type operators based on recurrent symmetric stable processes. Trans. Amer. Math. Soc. 368 (2016), 149-167.

[38] N. Torki-Hamza, Laplaciens de graphes infinis (I-graphes) métriquement complets. Confluentes Math. 2 (2010), 333-350.

[39] W. Veech, The necessity of Harris' condition for the existence of a stationary measure. Proc. Amer. Math. Soc. 14 (1963), 856-860.

[40] D. Vere-Jones, Ergodic properties of nonnegative matrices. I. Pacific J. Math. 22 (1967), 361-386.

[41] D. Windisch, Entropy of random walk range on uniformly transient and on uniformly recurrent graphs. Electron. J. Probab. 15 (2010), 1143-1160.

[42] W. Woess, Random Walks on Infinite Graphs and Groups, Cambridge Tracts in Mathematics 138, Cambridge University Press, Cambridge, 2000.

[43] R. K. Wojciechowski, Stochastic completeness of graphs. Thesis (Ph.D.)-City University of New York, ProQuest LLC, Ann Arbor, MI. 2008. 